\title{
Effective Capacity in Wireless Networks: A Comprehensive Survey
}

\author{
Muhammad Amjad, Leila Musavian, and Mubashir Husain Rehmani
}

\begin{abstract}
Low latency applications, such as multimedia communications, autonomous vehicles, and Tactile Internet are the emerging applications for next-generation wireless networks, such as 5th generation (5G) mobile networks. Existing physicallayer channel models, however, do not explicitly consider qualityof-service (QoS) aware related parameters under specific delay constraints. To investigate the performance of low-latency applications in future networks, a new mathematical framework is needed. Effective capacity (EC), which is a link-layer channel model with QoS-awareness, can be used to investigate the performance of wireless networks under certain statistical delay constraints. In this paper, we provide a comprehensive survey on existing works, that use the EC model in various wireless networks. We summarize the work related to $\mathrm{EC}$ for different networks such as cognitive radio networks (CRNs), cellular networks, relay networks, adhoc networks, and mesh networks. We explore five case studies encompassing EC operation with different design and architectural requirements. We survey various delay-sensitive applications such as voice and video with their EC analysis under certain delay constraints. We finally present the future research directions with open issues covering EC maximization.
\end{abstract}

Index Terms-Effective capacity (EC), quality-of-service, fading channels, delay constraints, real-time applications, wireless channel model, channel capacity.

\section{INTRODUCTION}

\section{A. Motivation: Need of Effective Capacity Mathematical Model in Wireless Communications}

Advances in wireless communications have resulted into emergence of a wide range of applications. Emerging wireless networks with advanced technologies such as full-duplex (FD) communications, non-orthogonal multiple access (NOMA), multiple input and multiple output (MIMO) and millimeter wave (mmWave) promise higher data rates [1]-[3]. With provision of this higher data rate and seamless connectivity, multimedia applications, which are regarded as delay-sensitive applications, have gained a lot of attraction [4]. This requires an efficient modeling of wireless channel that can take into consideration QoS metrics such as delay-violation probability, data rate, and end-to-end delay [5].

Packet switched networks can be analysed with the help of physical and link-layer channel models depicted in Figure 1. Using physical-layer channel models for analysing the

Please direct correspondence to M. Amjad.

M. Amjad and L. Musavian are with the School of Computer Science and Electronic Engineering, University of Essex, CO4 3SQ, UK (Email: m.amjad@essex.ac.uk, leila.musavian@essex.ac.uk)

M.H. Rehmani is with Department of Computer Science, Cork Institute of Technology, Rossa Avenue, Bishopstown, Cork, Ireland. (Email:mshrehmani@gmail.com). performance of delay-limited applications can be complex and inaccurate in some cases [6]. Hence, a new link-layer channel model named as "effective capacity (EC)" has been introduced [6]. With the help of EC, the channel can be modeled in terms of link-layer related QoS-metrics, such as probability of having non-empty buffer and delay violation probability. Concept of this link-layer channel model was first introduced in [6], which modeled a wireless link using two EC functions named as QoS exponent and probability of non-empty buffer. The developed link-layer channel model provides advantages such as ease of implementation and translation into the QoS guarantee, i.e., delay violation probability. Main motivations involving EC metric for various performance evaluations are highlighted below:

- EC modelling is based on an in-depth queueing analysis which can be used to characterize a relation between the source rate and the service rate taking into consideration both link-layer and physical layer parameters. Through this characterization, advance validation of communications systems performance such as efficient admission control can be achieved [7].

- EC is the dual concept of effective bandwidth [8], [9] and shows the maximum constant arrival rate for a wireless channel while satisfying a delay outage probability constraint [10]. This feature can be exploited to achieve the required QoS for some applications with specific QoS requirements.

- With the help of the EC concept, QoS provisioning over wireless links and efficient bandwidth allocation can be achieved in closed-form while satisfying certain delay guarantee constraints [6].

- The EC performance of well-known physical layer-based resource allocation algorithms, e.g, water filling, can be investigated. Performance of various proposed adaptive modulation and coding (AMC) schemes ${ }^{1}$ can be tested by using the EC metric [11].

- Using the EC model, the performance of adaptive resource allocation techniques for a specific QoS-aware connection can be analysed in closed-form in various cases. This, in turn, will pave the way for designing efficient resource allocation algorithms, hence improving the system performance.

- Provision of QoS guarantee with support for a variety of traffic flows requires efficient scheduling techniques. Using the EC concept, efficient delay constrained scheduling approaches can be designed [12].

${ }^{1}$ For further details, see Sections V and VIII. 


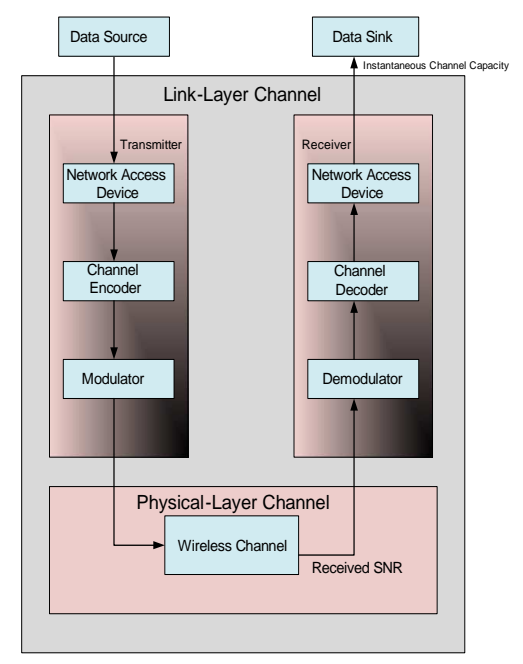

Fig. 1. Basic components involved in the communications of packet switched networks [6]. In this packet-based communications system, different components of physical and link-layer have been illustrated which shows the difference between physical and link-layer channel modelling.

\section{B. Contribution of This Survey Article}

The concept of EC has been extensively used in literature to test the performance of various delay-constrained wireless networks. However, to the best of our knowledge, there exist no comprehensive survey that can encompass the state-of-theart work of the EC model. This survey paper will be a first attempt to provide a comprehensive view of the EC model in wireless communications systems. In summary, following are the core contributions of our work:

- We cover a broad description of various applications for which their performance can be analysed using the EC model.

- We discuss five case studies that highlight the use of EC in five different wireless networks.

- We survey the achievable EC for various types of fading Models.

- We survey the achievable EC of cognitive radio networks, wireless local area networks, mesh networks, cellular networks, and full-duplex (FD) communications.

- We outline future research directions and open issues related to our survey, i.e., how the concept of EC can be used to analyze the performance of various wireless networks, and how this concept can be used for proposing efficient resource allocation and transmission designs.

\section{Review of Related Survey Articles}

As far as we can determine, there exists no comprehensive survey, that covers EC studies in various wireless networks. State-of-the-art work using the EC model has been performed with various fading conditions, supported applications, antenna designs, employed networks, retransmission schemes, etc. Most of the work on EC only covers one or more aspects of communications. There exists very limited work that covers multiple aspects of EC regarding the provision of statistical QoS in wireless communications. Authors in [13], have discussed various potentials and challenges that are associated
TABLE I

LIST OF ACRONYMS AND CORRESPONDING DEFINITIONS.

\begin{tabular}{|l|l|}
\hline Acronyms & Definitions \\
\hline 5G & Fifth Generation \\
\hline AF & Amplify-and-Forward \\
\hline AMC & Adaptive Modulation and Coding \\
\hline ARQ & Automatic Repeat reQuest \\
\hline AWGN & Additive White Gaussian Noise \\
\hline BER & Bit Error Rate \\
\hline BS & Base Station \\
\hline CDMA & Code-Division Multiple Access \\
\hline CR & Cognitive Radio \\
\hline CRN & Cognitive Radio Networks \\
\hline CSI & Channel State Information \\
\hline DF & Decode-and-Forward \\
\hline DSA & Dynamic Spectrum Access \\
\hline EC & Effective Capacity \\
\hline FIFO & Ftrst-In-First-Out \\
\hline FDCRNs & Full-Duplex Cognitive Radio Networks \\
\hline HARQ & Hybrid Automatic Repeat reQuest \\
\hline IP & Internet Protocol \\
\hline LLC & Logical Link Control \\
\hline LTE & Long-Term Evolution \\
\hline MAC & Media Access Control address \\
\hline MIMO & Multiple-Input and Multiple-Output \\
\hline MS & Mobile Stations \\
\hline NC & Network Coding \\
\hline OFDMA & $\begin{array}{l}\text { Orthogonal Frequency-Division Multi- } \\
\text { ple Access }\end{array}$ \\
\hline PU & Primary User \\
\hline PLR & Packet Loss Ratio \\
\hline QoE & Quality of Experience \\
\hline QoS & Quality of Service \\
\hline SINR & Signal-to-Interference-plus-Noise Ratio \\
\hline SDR & Software-Defined Radio \\
\hline SUs & Secondary Users \\
\hline SNR & Signal-to-Noise Ratio \\
\hline TCP & Transmission Control Protocol \\
\hline TDMA & Time-Division Multiple Access \\
\hline TVWS & TV White Spaces \\
\hline VANETs & Vehicular Ad hoc Networks \\
\hline VoD & Video on Demand \\
\hline WLAN & Wireless Local Area Networks \\
\hline WRANs & Wireless Regional Networks \\
\hline WSNs & \\
\hline ZFBF & \\
\hline
\end{tabular}


with the provisioning of statistical QoS requirements in bufferaided relay communication systems. Using the concept of EC, a trade-off of statistical delay between two concatenated queues has been discussed. This study focuses on one and two hops relay systems and summarizes the performance in terms of achievable rate and secrecy rate. The survey also covers the performance of caching in delay QoS-aware relay systems. Other wireless networks such as cognitive radio networks (CRNs), cellular, mesh, and ad hoc have not been considered with various fading models.

Further, EC of multiple antenna systems (distributed and co-located) has been discussed in [14]. MIMO systems have been analyzed while the establishing the EC perspective and performance comparison of distributed and co-located antenna systems. This study is a good contribution related to the EC analysis of large scale MIMO systems. However, [14] does not cover in much detail the EC-based performance evaluation of wireless communications. While EC characterization of multiple antenna systems has been discussed for mobile networks in [14], the EC perspective of other advanced and emerging wireless networks has not been considered. On another note, the EC-based performance evaluation of CRNs while considering channel sensing and spectrum management paradigm in a dynamic spectrum access environment has been discussed in [15] wherein an EC-based model for assessing the performance of CRNs with various PUs activity patterns has been developed. While this work discusses the basis for analysing EC in CRNs, it only focuses on CRNs with various spectrum sensing features.

We note that an extensive work has been done on EC-based performance evaluation of different communications networks but a comprehensive survey is yet to be conducted on this topic. This has motivated us to present a comprehensive survey of the cross-layer EC model that can be used to test the delaylimited performance of various wireless networks.

\section{Article Structure}

Table I shows different acronyms that has been used in the article. The rest of the article is organized as follows: Section II presents the basic definitions of EC and effectivebandwidth. Section III covers the five case studies (in five networks) that employ the EC model, and in Section IV, various delay-constrained applications for which their performance is tested through EC have been surveyed. Classification of various fading models with their corresponding achievable EC has been provided in Section V, while in Section VI, different wireless networks with the EC concept has been discussed in detail. Section VII discusses the achievable EC in FD communications and Section VIII covers the various retransmission schemes that have been analyzed with the EC concept. Open issues and future research directions have been discussed in Section IX. The entire article has been concluded in Section X.

\section{ThEORY OF EFFECTIVE CAPACITY}

In this section, a basic overview of EC has been provided. We note that EC is the dual concept of effective-bandwidth as presented in the pioneer work of Dapeng $\mathrm{Wu}$ in [6]. Hence, in order to have a good understanding of EC, we first introduce the concept of effective bandwidth.

\section{A. Effective Bandwidth}

The concept of effective-bandwidth is derived through the large-deviation principle and can show the minimum constant service rate that is needed to satisfy a given queueing delay requirement for a given source rate [16]. Effective bandwidth has been used extensively for obtaining optimal resource allocation schemes. Since effective bandwidth is based on large-deviation principle, it is traditionally used in systems with large delay bounds. However, effective bandwidth has also been recently used in scenarios where delay bound is short. For example, in [17], the concept of effective bandwidth is used to design an adaptive resource allocation scheme for a system with ultra low latency requirements. Below is the description of the effective-bandwidth approximation:

Let us consider a first-in-first-out (FIFO) queue with packets arrival rate at $t$ as $\mu(t)$, the number of packets in the queue as $q(t)$, capacity of the link at time $t$ as $c(t)$ and an infinite buffer size. We consider $q(t)$ to converge to a steady state $q(\infty)$ and define $A\left(t_{1}, t_{2}\right)=\sum_{t=t_{1}+1}^{t_{2}} \mu(t)$ as the total number of arrivals at $\left(t_{1}, t_{2}\right]$ and $C\left(t_{1}, t_{2}\right)=\sum_{t=t_{1}+1}^{t_{2}} c(t)$.

Authors in [18], [19] have proposed a theorem to derive the theory of effective bandwidth. For this purpose, the following assumptions are used as presented in [18], [19].

Let assume

- Arrival rate $\mu(t)$ and the service rate $c(t)$ are both ergodic and stationary. Also $\mathbb{E}[\mu(t)]<\mathbb{E}[c(t)]$, where $\mathbb{E}[$.$] shows$ the expectation operator.

- Arrival rate and source rate $(\mu(t)$ and $c(t))$ are independent.

- Using the Gartner-Ellis theorem, we have for all $\theta \in$ $\mathbb{R}, \Lambda_{A}(\theta) \triangleq \lim _{t \rightarrow \infty} \frac{1}{t} \log \left(\mathbb{E}\left[e^{\theta A(0, t)}\right]\right)$ and $\Lambda_{C}(\theta) \triangleq$ $\lim _{t \rightarrow \infty} \frac{1}{t} \log \left(\mathbb{E}\left[e^{\theta C(0, t)}\right]\right)$, where $\theta$ is the delay exponent, and $\Lambda_{A}(\theta)$ and $\Lambda_{C}(\theta)$ are assumed differentiable.

Now if there exists a unique $\theta^{*}>0$, such that the below equation holds between the effective bandwidth and EC:

$$
\Lambda_{A}\left(\theta^{*}\right)+\Lambda_{C}\left(-\theta^{*}\right)=0,
$$

then, mathematical derivations are provided in [18], [19] that relates the value of $\theta^{*}$ (found from (1)) to the buffer overflow probability according to

$$
\lim _{x \rightarrow \infty} \frac{\log (\operatorname{Pr}\{q(\infty) \geq x\})}{x}=-\theta^{*},
$$

where $\operatorname{Pr}\{a \geq b\}$ shows the probability of $a$ being greater than or equal to $b$.

Proof. For proof please refer to [18], [19].

Let $x$ be the buffer size of the link. Packets are usually dropped when buffer becomes full. From (2) packet loss probability $\epsilon$ can be approximated as [20]

$$
\epsilon=e^{-\theta^{*} x}
$$

Assuming that the link has constant capacity such that $c(t)=c$, for all $t, \Lambda_{C}\left(-\theta^{*}\right)$ can be simiplied as 


$$
\Lambda_{C}\left(-\theta^{*}\right)=\lim _{t \rightarrow \infty} \frac{1}{t} \log \left(e^{-\theta^{*} c t}\right)=-\theta^{*} c .
$$

Using (1), we get $\frac{\Lambda_{A}\left(\theta^{*}\right)}{\theta^{*}}=c$. To have a small packet loss probability, a capacity of the link equal to $\frac{\Lambda_{A}\left(\theta^{*}\right)}{\theta^{*}}$ is required where the value for $\theta^{*}$ comes from the unique solution of $\theta^{*}=-(\log \epsilon) / x$ (derived from 3 ).

\section{B. Effective Capacity}

Authors in [6] introduced the concept of EC by taking motivations from the theory of effective bandwidth. EC is the dual concept of effective bandwidth. Recall that effective bandwidth shows the minimum service rate that is needed to guarantee a delay requirement for a given source traffic. The EC model, on the other hand, can be used to find the maximum source rate that the channel can handle (service rate) with the required delay constraint. As has been discussed above, the concept of EC can be used when a delay bound is large. However, it can also be used to test the performance of a system when delay bound is small, as has been discussed in [17]. Analytical framework for deriving EC has been discussed briefly below:

We assume the service process $c(t), t=0,1,2, .$. , with a partial sum $C(t 1, t 2)=\sum_{t=t_{1}+1}^{t_{2}} c(t)$ is ergodic and stationary. Further, the Gartner-Ellis limits for this service process is expressed as

$$
\Lambda_{C}(\theta) \triangleq \lim _{t \rightarrow \infty} \frac{1}{t} \log \left(\mathbb{E}\left[e^{\theta C(0, t)}\right]\right) .
$$

From (1), we get

$$
E_{c}\left(\theta^{*}\right)=-\frac{\Lambda_{C}\left(-\theta^{*}\right)}{\theta^{*}}=\mu .
$$

We recall that (6) is EC of service process, while $\theta^{*}$ is the QoS exponent. The delay outage probability can now be formulated as

$$
\lim _{x \rightarrow \infty} \frac{\log (\operatorname{Pr}\{q(\infty) \geq x\})}{x}=-\theta^{*} .
$$

A more stringent QoS requirements can be represented by a larger value of $\theta^{*}$ with a faster decay rate. However, smaller values of $\theta^{*}$ represent slower decay rates and provide looser QoS guarantees.

Now, an expression for the delay $(D(t))$ experienced by a packet at any time $t$ can also be approximated as follows

$$
\operatorname{Pr}\left\{D(t)>D_{\max }\right\} \approx \operatorname{Pr}\{q(\infty)>0\} e^{-\theta^{*} \mu D_{\max }},
$$

where $\operatorname{Pr}\{q(\infty)>0\}$ is the probability of non-empty buffer and $D_{\max }$ is the maximum delay bound. EC is the combination of two functions, namely, QoS exponent and probability of non-empty buffer.

The probability of non-empty buffer can be achieved by considering the

$$
\operatorname{Pr}\{q(\infty)>0\} \approx \frac{\mathbb{E}[\mu(t)]}{\mathbb{E}[c(t)]}
$$

The above analytical explanation of effective-bandwidth and EC can be summarized as follows:
- The value of EC at $\theta^{*}, E_{c}\left(\theta^{*}\right)$, shows the maximum constant arrival rate. Hence, $\left.\mu \leq E_{c}\left(\theta^{*}\right)\right)$ should hold.

- The solution for $\theta^{*}$ can be found when $E_{b}\left(\theta^{*}\right)=E_{c}\left(\theta^{*}\right)$ (for the arrival and source processes) holds.

- Using (9), the probability of non-empty buffer can be estimated.

- Using (8), the delay-violation probability can be estimated by using the pre-determined value of delay bound, probability of non-empty buffer, and obtained value of $\theta^{*}$.

\section{Case Studies Involving EfFective Capacity MEASURES}

To illustrate an in-depth understanding of the EC metric and how it can be used to analyze the performance of different network architectures or application scenarios, five case studies have been presented. These include device-to-device (D2D) communications, cellular networks, full-duplex communications, peer-to-peer communications, and visible light communications. These case studies show the performance of various networks when handling delay-sensitive multimedia applications by using EC concept. The motivation of providing these case studies is to show the broad use of the EC modelling. In fact, EC can be used to test the performance of diverse network topologies, resource allocation schemes, traffic characterization, and various admission control policies. Brief discussion of each case study has been described below:

\section{A. Cellular Communications}

EC-based delay analysis while considering cellular communications has been well investigated in literature [21]-[25]. QoS-aware real-time and delay-sensitive applications have been evaluated using the EC metric with different channel conditions and imperfections. In [26], the main architecture of cellular network involving the EC model with one mobile station (MS) and a base station (BS) is considered. In this cellular network, resources have been allocated based on QoSconstraints using the EC model. A queueing behavior at datalink layer has been analysed by investigating the maximum achievable EC. The EC model has been used either at MS or BS to evaluate the performance of the network when handling a QoS-aware traffic. Moreover, the QoS constraints are categorized either homogeneous or heterogeneous, depending on the known symmetrical and asymmetrical EC regions. This known EC regions show the impact of delay exponent in shaping the QoS constraints for two and three MS cases.

\section{B. Peer-to-Peer Video Streaming}

In peer-to-peer streaming, the EC model has been used to analyze the network performance. In detail, to efficiently analyse the peer-to-peer streaming, authors in [27] have incorporated the concept of an EC peer-selection (ECPS) approach for mobile users. In the proposed approach, mobile users can enjoy efficient video streaming without facing long delay. In this peer-to-peer streaming, multiple attribute decision making 
(MADM) approaches have been used to accommodate various factors such as power-level, signal-to-interference and noise ratio (SINR) and mobility of peers.

\section{Visible Light Communications}

Using the visible light, which is between 400 and $800 \mathrm{THz}$ band, as a communication medium for next generation wireless networks promises enhanced data rate for many delay-sensitive applications [28].

Usage of multiple radio access schemes, such as millimeterwave, UMTS, WLAN, and visible light communications can also result into overlapping of their coverage area. This approach has resulted into the heterogeneous networks domain to cover various radio access technologies of next-generation networks. Most of the existing work on EC considers visible light communication scenario within the heterogeneous network architecture with cellular networks (such as femto cells as in [29]). Existing works on visible light communications usually take into consideration the EC concept to assess QoS-awareness in heterogeneous networks [30]. With the introduction of the EC model in the optical communications, satisfying the statistical delay requirements while supporting user-centric (UC) cluster formation has been investigated. The achievable EC and the sum utility functions are quantified for the UC cluster-formation. By applying the concept of EC and sum utility, the problem becomes tractable, and is solved by using the exhaustive search. This analysis shows that the UC cluster formation achieves higher EC compared to conventional cellular network designs.

\section{Full Duplex Communications}

With the advances in self-interference suppression (SIS) approaches, the dream of full-duplex (FD) communications has been realized ${ }^{2}$. With the advent of FD communications, simultaneous sending and receiving on the same spectral band can almost double the throughput as compared to halfduplex communications [31]. With enhanced data rate, FD communications has been extensively studied for multimedia applications with stringent delay requirements [32]. The EC model has also been used in FD communications to test the performance of the network for various QoS-aware applications [33]. However, the maximum achievable EC of many FD paradigms, such as, directionality, beamforming, and various transmit and receive antenna pairing schemes is not known yet. Most of the work on FD communications with consideration of EC has been presented with FD-relay networks. For example, the maximum constant arrival rate of an FD-enabled communication system while satisfying a predefined delay constraint is found in [32]. With the implementation of proper SIS approach, such as, passive or active SIS approach, the maximum achievable EC of an FD communication paradigm has been found in [34]. In this study, by finding the optimum fixed value for the source arrival rate (EC), the properties of the source and relay has been investiaged. Afterwards, the depending on the achievable EC of the source, the optimal resource allocation for the relay and source are derived.

\footnotetext{
${ }^{2}$ for detail on FD and SIS approaches see Section VII.
}

\section{E. Summary and Insights}

In this section, five case studies encompassing the concept of EC has been showcased to understand a wide-range of applicability of the EC model ranging from cellular networks to visible light communications. Ensuring the QoS constraints in wireless networks that deal with delay-sensitive applications is in fact a challenging task. We note that, channel conditions determine the capacity of a network, and as such, whether or not the required QoS constraints is achievable. The variability in wireless channels results in variability in the transmission buffer status and, in turn, in the experienced delay by the transmitted packets. The concept of EC, that takes into consideration the physical layer parameters in conjunction with the link-layer and provides a simple formulation for a link-layer performance is well received by the researchers working in various networks. This concept, not only can be used for analyzing the performance of the networks, but it also provides a strong mathematical framework for efficient design of the system parameters. This section is clear description of a wide applicability of the EC model in traditional, as well as, emerging wireless networks such as URLLC and visible light communications.

\section{VARIOUS QOS-AWARE APpliCATIONS ANALYSED WITH THE EC MODEL}

In this section, we provide an in-depth analysis of various applications using the EC model. Table II presents the details of various applications which are specifically analysed with the concept of EC in various networks. Different applications have been classified into voice, video, and miscellaneous applications. This classification is based on the delay requirements and network architecture used with respect to different applications.

\section{A. Voice Applications}

Plethora of applications can be tested with EC, while taking into consideration certain QoS requirements [85]. We have further classified the voice applications into voice call, VoIP, and cellular telephony. Different voice applications are classified based on different communication paradigm used such as voice over Internet protocol or traditional wireless networks. Details of each sub-category have been presented below:

1) Voice Calls: Idea of a link-layer channel model with QoS-aware metric support has been used in various networks with different architectures to assess the quality of voice calls. Compared to video calls, voice calls are regarded as delay-sensitive low data rate applications [35]. To analyse the quality of voice calls with small delay, EC-based source traffic and service characterization can be carried out. In [22], the EC model has been used to analyse the performance of the proposed, but not necessarily perfect, design methods to support voice applications. Four antennas have been used, and achievable EC was obtained to test the delay-limited performance of multiple antenna systems for voice applications. This multi-antenna based EC analysis shows that $90 \%$ of the system performance or voice quality is reduced due to 
TABLE II

DIFFERENT DELAY-SENSITIVE APPLICATIONS INVOLVING EFFECTIVE CAPACITY MEASUREMENTS

\begin{tabular}{|c|c|c|c|c|}
\hline \multicolumn{2}{|l|}{ Different Applications } & Architecture or Network Used & Papers & Fading Channel Used \\
\hline \multirow[t]{13}{*}{ Voice Applications } & \multirow[t]{7}{*}{ Voice Calls } & Cellular Network & {$[22]$} & Rician Fading Channels \\
\hline & & OFDMA-Based Networks & {$[35]$} & Rayleigh Fading Channels \\
\hline & & Multi-hop Networks & [36] & Not Defined \\
\hline & & Cognitive Radio Networks & {$[37]$} & Rayleigh Fading Channels \\
\hline & & Multi-hop Networks & {$[38]$} & Not Defined \\
\hline & & Cross-layer Network Design & {$[39]$} & Nakagami- $m$ Fading Channels \\
\hline & & Proactive Link Selection Routing & {$[40]$} & Not Defined \\
\hline & \multirow[t]{5}{*}{ VoIP Applications } & Long Term Evolution & [41] & Rayleigh Fading Channels \\
\hline & & Multi-user Network Layout & {$[42]$} & Rician Fading Channels \\
\hline & & Wireless Sensor Networks & [43] & Rayleigh Fading Channels \\
\hline & & Cognitive Radio Networks & [44] & Rayleigh Fading Channels \\
\hline & & Relay Networks & {$[45]$} & Rician Fading Channels \\
\hline & Cellular Telephony & Cellular Networks & [6] & Rayleigh Fading Channels \\
\hline \multirow[t]{3}{*}{ Miscellaneous applications } & Medical Application & WiMAX Networks & [46] & Not Defined \\
\hline & Smart Grid Application & Non-Intrusive Application & [47] & Rician Fading Channels \\
\hline & Image Transmission & Multi-hop Mesh Networks & [48] & Rayleigh Fading Channels \\
\hline \multirow[t]{41}{*}{ Video Applications } & \multirow[t]{33}{*}{ Video Streaming } & Mobile Networks & {$[39]$} & Nakagami- $m$ Fading Channels \\
\hline & & Cross-layer Design & [49] & Rayleigh Fading Channels \\
\hline & & Long Term Evolution & [50] & EPA Fading Channels \\
\hline & & Cross-layer Design & {$[51]$} & Rayleigh Fading Channels \\
\hline & & Wireless Cooperative Networks & {$[52]$} & Generalized $k$ Fading Channels \\
\hline & & Multi-user Video Streaming & [53] & Correlated Fading Channels \\
\hline & & Multi-Channel & {$[54]$} & Rayleigh Fading Channels \\
\hline & & Broadband-ISDN & $\frac{155]}{[55]}$ & Not Considered \\
\hline & & Multi-user Video Streaming & $\frac{156]}{[56]}$ & Rayleigh Fading Channels \\
\hline & & Wireless Local Area Networks & [57] & Nakagami- $m$ Fading Channels \\
\hline & & 5G Networks & [58] & Nakagami- $m$ Fading Channels \\
\hline & & FD-Relay Networks & [59] & Rayleigh Fading Channels \\
\hline & & Cognitive Radio Networks & [60] & Nakagami- $m$ Fading Channels \\
\hline & & $5 \mathrm{G}$ Networks & [61] & Nakagami- $m$ Fading Channels \\
\hline & & FD-Relay Networks & {$[62]$} & Nakagami- $m$ Fading Channels \\
\hline & & Femto Cells & {$[63]$} & Not Considered \\
\hline & & Wireless Local Area Networks & {$[64]$} & DTMC-Based Fading Channels \\
\hline & & OFDMA-Based Networks & [65] & Nakagami- $m$ Fading Channels \\
\hline & & Wireless Local Area Networks & [66] & Rayleigh Fading Channels \\
\hline & & Multi-User Video Streaming & [67] & Rayleigh Fading Channels \\
\hline & & Multi-User Video Streaming & [68] & Rayleigh Fading Channels \\
\hline & & Cross-Layer Design & [69] & Rayleigh Fading Channels \\
\hline & & WiMAX & [70] & Rician Fading Channels \\
\hline & & Multi-User Video Streaming & [71] & Not Defined \\
\hline & & Wireless Virtual Networks & {$[72]$} & Rayleigh Fading Channels \\
\hline & & OFDMA-Based Networks & [73] & Rayleigh Fading Channels \\
\hline & & Wireless Sensor Networks & [74] & Rayleigh Fading Channels \\
\hline & & Multi-User Video Streaming & [75] & Rayleigh Fading Channels \\
\hline & & Wireless Local Area Networks & {$[76]$} & Not Defined \\
\hline & & Cross-Layer Network Design & [77] & Nakagami- $m$ Fading Channels \\
\hline & & Heterogeneous Wireless Networks & {$[78]$} & Not Defined \\
\hline & & Cellular Networks & {$[79]$} & Rayleigh Fading Channels \\
\hline & & Single User Video application & [80] & Rayleigh Fading Channels \\
\hline & \multirow[t]{3}{*}{$\begin{array}{l}\text { High Speed Video Transmis- } \\
\text { sion }\end{array}$} & Secure Wireless Networks & {$[81]$} & Nakagami- $m$ Fading Channels \\
\hline & & Multi-User Video Transmission & [82] & DTMC-Based Fading Channels \\
\hline & & Two-Hop Networks & [83] & Rayleigh Fading Channels \\
\hline & \multirow[t]{3}{*}{ Light Video Transmission } & Secure Wireless Networks & {$[81]$} & Nakagami- $m$ Fading Channels \\
\hline & & Multi-user Video Transmission & {$[82]$} & DTMC-Based Fading Channels \\
\hline & & Two-Hop Networks & [83] & Rayleigh Fading Channels \\
\hline & Peer-to-Peer Streaming & Peer-to-Peer Networks & {$[27]$} & Not Defined \\
\hline & Video Conferencing & Multi-User Video Transmission & {$[84]$} & Rayleigh Fading Channels \\
\hline
\end{tabular}

imperfections in the system. This framework was also used to investigate the enabling technologies such as W-WCDMA and MC CDMA for the fourth generation wireless networks. Various quality constraints for voice calls with EC as the performance metric have been investigated in [36], [38]. In this study, issue of service degradation and source dissatisfaction has been tested using EC metric while proposing an optimal resource allocation scheme.

An optimal resource allocation scheme, using the EC model, for voice calls in CRNs has been presented in [37]. Using EC, an optimal sensing time and channel allocation scheme has been analysed in detail. The proposed resource allocation scheme has also been evaluated through extensive simulations to show its effectiveness. Cross-layer EC modeling for testing performance of QoS-aware applications such as independent and identically distributed (i.i.d.) and non i.i.d fading channels were estimated while taking into consideration the multicast receivers was studied in [39]. Extensive simulations were also performed to clearly demonstrate the trade-off between different QoS metrics. The EC model to test the quality of voice and other delay applications has also been presented with unicast routing control agent (URCA) in [40]. The proposed routing scheme can evenly distribute the load over all the available paths, and minimize the impact of link failure on the performance of network. From the achieveable EC of the individual links, the soft link failures are predicted. The soft link failures are then minimized to ensure the success of critical voice sessions. 
2) VoIP Applications: With the advancement in Internet protocol (IP)-based networking, voice over Internet protocol (VoIP) applications have gained a lot of attention. The maximum achievable EC of VoIP or IP telephony has been extensively studied and analysed while considering various network and architectural designs under different fading channels in [41]. In [42], achievable EC of wireless networks with multiple input single output (MISO) for VoIP applications has been investigated in detail. As compared to the traditional work that considers Rayleigh fading, this work considers the provision of statistical QoS-guarantee under Rician fading channels. With the help of EC concept, effective rate that can support future applications like VoIP, has been measured . A routing protocol that takes into consideration the end-to-end delay for wireless sensor networks has been proposed in [43]. This routing scheme is then investigated with the help of the EC concept to find the shortest possible paths while residing within the required delay constraints. This routing scheme is then tested with the VoIP applications.

In addition to WSNs, VoIP applications have also been investigated in CRNs with the concept of EC [44]. In particular, an EC-based optimal resource allocation scheme for cognitive radio (CR)-based femto cells have been investigated. This resource allocation scheme also takes into consideration the cross-tier interference, and hence, provides a significant support for delay-sensitive applications such as VoIP. VoIP applications support in emerging futuristic networks such as mobile multi-hop networks with the EC model has also been discussed in [45]. In this study, authors have investigated the achievable EC of multi-hop mobile networks and then assess the network functionality with different delay-bounds. In this work, a cross-layer simulation platform has also been proposed to study the impact of various delay-sensitive applications such as VoIP on multi-hop network model.

3) Cellular Telephony: Simplest of the voice applications is the traditional cellular telephony. In [6], authors have used the concept of EC to investigate the QoS in for a simple scenario of cellular telephony. In this study, a comparative view of physical layer channel model and link-layer channel model (EC) has been investigated. Cellular telephony has been tested by physical as well as by EC parameters. With the help of two EC functions, a wireless link has been modeled to provide the QoS-guarantee for the delay-sensitive traffic. This study is the pioneering work on the EC concept based on the idea of effective bandwidth. This was the first attempt to investigate the link-layer channel model while taking into consideration the statistical QoS provisioning in wireless networks.

\section{B. Miscellaneous Applications}

Existing work on the EC model in wireless networks can also take into consideration other low latency and real-time applications such as 2D, monitoring, medical, and smart grid applications [48], [86], [87]. Below is the description of various applications that require low latency and that have been discussed in conjunction with the EC concept in wireless networks.
1) Medical Applications: The EC model has been used to test the performance of the system for certain advance medical applications. In [46], authors have investigated end-toend delay distributions for tele-ultrasonography based on the EC modeling of wireless channels. For this purpose, a crosslayer simulation platform that consists of a source of medical ultrasound at a remote location has been analyzed over the WiMAX link. Extensive simulations show the effectiveness of the proposed scheme.

2) Smart Grid Applications: Traditional power grid has been transformed into the intelligent smart grid [88], [89]. Smart grid generates different types of multimedia traffic and $\mathrm{CR}$ is considered to be a potential technology which can assist multimedia applications in smart grid environment [90], [91]. In [47], authors have used the EC model to measure quantitatively the support of various smart grid multimedia applications in existing wireless communications designs. Different case studies considering various smart grid multimedia applications with their implementation in various communication scenarios with the EC model has been discussed in detail. In this case, EC comes out to be an efficient tool to quantify the performance of various smart grid applications regardless of different network technologies.

\section{Video Applications}

An extensive work on achievable EC in wireless networks considers video applications as the test applications to assess the various delay requirements of a system [27], [53]-[55]. In this classification, most of the studies consider delay-sensitive video applications using different video codecs. In comparison to the voice applications, various network designs with video transmission capabilities have been investigated with their achievable EC.

Video applications are regarded as delay-sensitive and timecritical application, that require QoS-guarantees. In this subsection, an overview of various video applications such as video streaming and video conferencing has been presented with the EC model.

1) Video Streaming: Video streaming is a challenging application due to its strict delay bound and bursty flow. With these limitations, transmission of videos over wireless medium with stringent delay requirements seems to be a challenging task [72]. In this respect, the EC model can be used for assessing the performance of various low latency applications with stringent QoS requirement while residing within a given delay violation probability.

Indeed an extensive work has been done on EC modelling of wireless channel while taking into account the video streaming applications [39], [49]-[52], [56]-[71], [73]-[80]. In the above mentioned works, authors have used the EC model in different fading conditions, such as Rayleigh, Nakagami- $m$, and Rician. However, most of the works of the EC model with video related applications consider Rayleigh fading channels. Only the works in [39], [57], [60]-[62], [65], [77] consider Nakagami$m$ fading channels with the EC model. Video streaming support have also been investigated with EC concept while using Rician fading channels [70]. In this study, the achievable 
EC has been studied with the physical layer IEEE 802.162004 WiMAX standard. By using the video streaming scenario with different channel conditions, different delay values are estimated to gaurantee the uninterrupted video quality.

Video surveillance or monitoring applications were also investigated with the EC model in [92]. In this work, concept of EC has been used to forsee the QoS guarantee in cognitive relay networks. In this work, the performance of a cognitive relay (COR) algorithm to support efficient video surveillance has been analyzed in terms of its achievable EC. This COR approach has also been explored for machine-to-machine (M2M) communications.

2) High/Low Speed Video Transmission: To simplify the classification of different video applications depending on the delay requirements, we can also categorize them into high and low speed. For example, the video-conferencing and online gaming with stringent delay requirements can be categorized as high speed video transmission. Other applications such as traditional video-streaming with less stringent delay and date rate requirements can be classified as low-speed video transmission.

High speed video transmission demands more control and resources as compared to the traditional video streaming applications [81]. Authors in [83], have provided a QoSaware power allocation based on achievable EC over two hop networks. This resource allocation scheme is then utilized to support high and low speed video transmission. Total power consumption is also minimized while guaranteeing the specific QoS requirements. In comparison to single hop networks, this study focuses on two hop DF relaying transmissions. Using the EC metric, delay distributions of two hops are obtained. In order to provide statistical-delay gaurantees for two hops relays, it is estimated that the delay distributions of both hops should be same, which then is achieved with the help of an asymmetric resource allocation scheme.

3) Video Conferencing: Performance of advanced real-time applications such as video conferencing has also been tested with EC metric. In [84], authors have obtained EC of multiple antenna systems which shows a significant EC gain using multiple antennas. Specifically, the achievable EC of a multiantenna Rayleigh fading channel with a procedure called channel hardening has been found. The overall gain achieved by using the EC model has been exploited to achieve smooth video conferencing without a large delay.

\section{Summary and Insights}

In this section, the performance of various real-time and delay-sensitive applications using EC model has been explored for different applications such as various design, and architecture. Delay limited performance analysis of QoSaware applications shows that data loss could occur when delay thresholds are violated and testing the performance of applications with stringent delay requirements are specifically more challenging. Indeed, it is a well-known fact that for applications with stringent-delay and ultra-reliability requirements, such as online gaming, video-conferencing, and autonomous vehicles, using the physical layer only parameters for capacity estimation may not be accurate. The suitability of this mathematical framework to model the performance of the network while residing within the stringent delay and reliability requirements need further research to be verified.

\section{EFFECTIVE CAPACITY ANALYSIS WITH DIFFERENT FADING MODELS}

In this section, we have provided a survey of existing work with their achievable EC with different fading models used in various wireless networks. Figure 2 show different fading models that have been taken into consideration with the EC concept. We note that channel variability can cause long delays in the transmission buffers. Hence, indicating the importance of using a suitable mathematical framework for testing the performance of the networks. The EC model can indeed be used in designing the adaptive resource allocation and scheduling schemes [230] that are specifically suitable for applications with constraints on the buffer size. The main advantages of utilizing the EC model with different fading models are the provision of a general mathematical framework and simplification of QoS-aware metrics.

\section{A. Stochastic Fading Models}

Stochastic fading models cover the fading in a channel that results from scattering and multipath propagation. In these models, a random variable is added to show the additional fading.

We recall that EC provides a generalized link-layer mathematical framework (its complexity has been discussed in Section V-E) for testing the performance of the channel under delay constraints. On that basis, different fading models can be analyzed with their distinct characters. Existing work on the EC model mostly take into consideration the stochastic fading models for an assessment of QoS-awareness in wireless networks. Among the stochastic fading models, Rayleigh and Nakagami- $m$ fading channels have been used extensively with EC concept. Current work in wireless networks considers different versions of stochastic fading models including Rayleigh, Nakagami- $m$, Rician, log-normal, and Weibull fading channels with EC metric. Below is the description of each fading channel:

1) Rayleigh Fading Channels: Most of the existing work on EC in wireless communications considers Rayleigh fading channels. Rayleigh fading is more prominent when there is no line of sight communications between the transmitter and receiver. Following works [6], [23], [26], [28], [29], [31], [32], [35], [41], [44], [49], [54], [66]-[69], [73]-[75], [79], [80], [83], [84], [132]-[218], [231] consider Rayleigh fading with the EC model in different wireless networks. More prominent wireless networks that have been investigated with Rayleigh fading channels with the EC model, are CRNs, cellular networks, and cooperative networks including the FD-relay networks. In cellular networks, with statistical QoS provisioning, Rayleigh fading has been extensively evaluated with EC metric. In CRNs with multiple channels, prediction related to multiple interference has also been studied with 


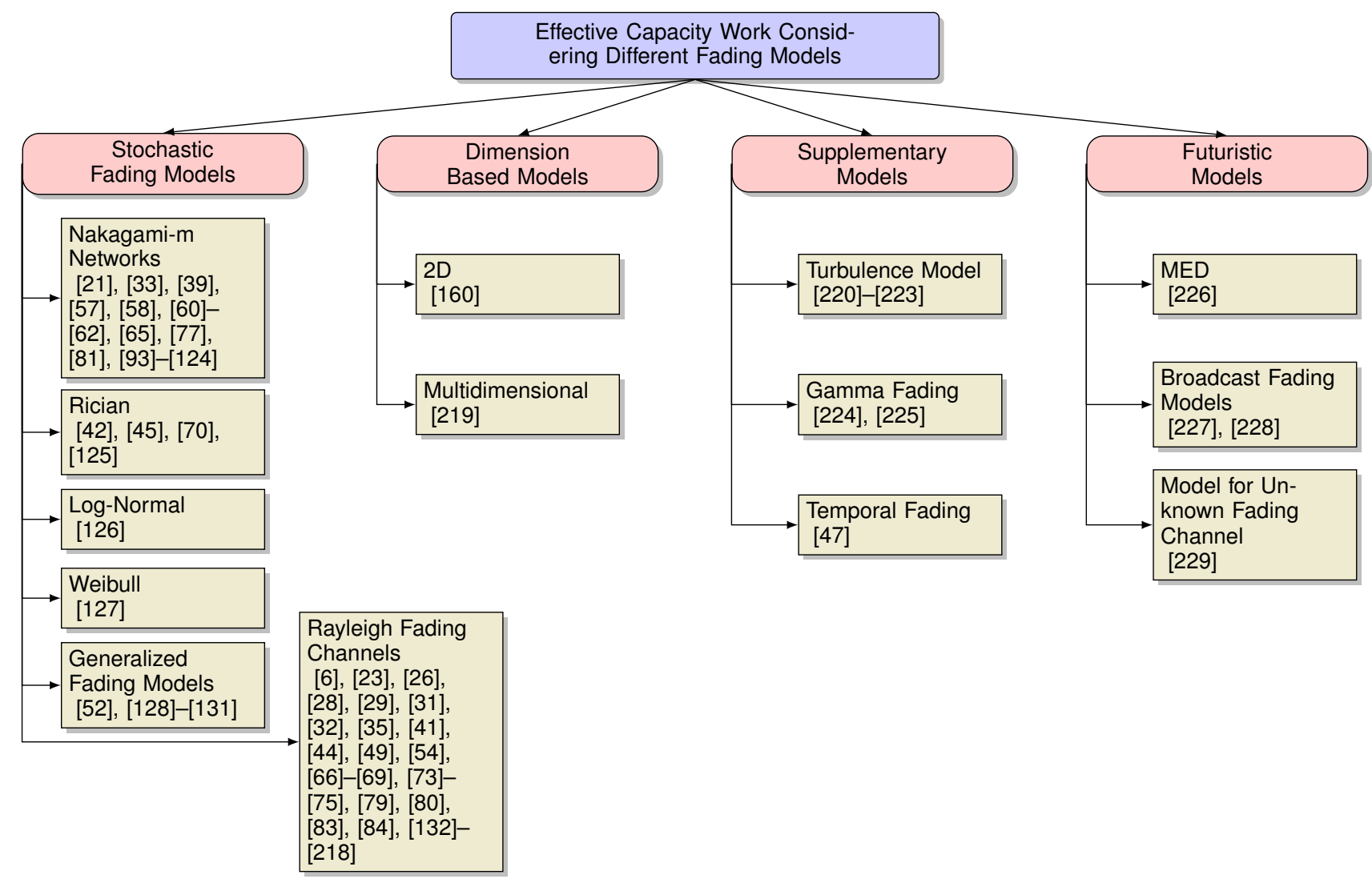

Fig. 2. The existing work on effective capacity with respect to different fading models can be classified in stochastic, generalized, futuristic, dimension-based, and supplementary fading models

Rayleigh fading channels. Achievable EC in CRNs with multiple channels and Rayleigh fading as the physical channel model has been extensively investigated to find the maximum arrival/source rate with the required delay-outage probability.

Most of the delay-sensitive applications with Rayleigh fading in wireless networks have also been evaluated with EC metric. Rayleigh fading has been used extensively because it helps the researchers to understand the radio signals in heavily urban environment. Closed-form expression of achievable EC with Rayleigh-fading is less complex as compared to the Nakagami- $m$ fading channel. Therefore, maximization in EC of different wireless networks with Rayleigh-fading has been investigated extensively in the existing works. Another fading channel, that has been used extensively after Rayleigh fading is Nakagami- $m$ fading channel.

2) Nakagami-m: For EC-based delay performance estimation of wireless networks, where the large delay-time spreads are going to be estimated, Nakagami- $m$ fading channels are used by clustering different reflected ways. Authors in [21], [33], [39], [57], [58], [60]-[62], [65], [77], [81], [93]-[124], have considered Nakagami- $m$ fading distributions in different wireless networks using the EC model. Nakagami- $m$ channel model is often regarded as the general fading channel and can be used to investigate the mobile and indoor-mobile scenarios. Depending upon the parameter $m$, where $m \in\left\{\frac{1}{2},+\infty\right\}$, different fading conditions can be achieved. For example, when $m=\frac{1}{2}$, this represents the severe fading case, while $m=1$ is the Rayleigh-fading, $m>1$ approximates the Rician channel, and $m=\infty$ represents additive white Gaussian noise (AWGN).

The main advantage of using Nakagami- $m$ fading distribution with the EC model in wireless networks is better matching of its empirical data as compared to other distributions such as Rayleigh and Rician. Authors in [111], have investigated the EC model with Nakagami- $m$ fading channel. This study reveals that uncorrelated Nakagami- $m$ flat fading conditions can well be analyzed with EC-based QoS-aware model. Complementary cumulative distribution function (CCDF) of delay has also been approximated by the EC model in this work. This analytical approximation based on EC leads to understanding the delay statistical behavior, which is not possible with the physical layer channel models.

Works of CRNs [98], [103], [115] and relay networks [100], [111] show that queueing behavior can well be evaluated with EC metric under Nakagami- $m$ fading conditions.

3) Rician: As compared to Rayleigh fading channel, in Rician fading, out of several different paths one must be the line of sight path. In this fading conditions, amplitude of the propagated signals are modelled by using Rician distributions. Achievable EC of wireless networks with Rician fading conditions has been discussed in [42], [45], [70], [125]. As Rician fading conditions consider one strong component, this strong component can be the line of sight path, therefore Rician fading can be employed in some advance networks such as satellite communications [232] which studies the EC model 
of channel. We note that the satellite communications suffer from long delays in signal transmission due to the very long distance between the satellite and the users. Hence, the concept of EC can be very useful for analyzing the performance of these communications systems. In addition to satellite communications, the EC model with Rician fading has also been studied in cellular communications, indoor networks, and vehicular networks.

4) Log-Normal: In addition to Rayleigh, Nakagami- $m$, and Rician fading conditions, other stochastic fading models such as log-normal has also been investigated with the EC model. In log-normal fading channel, the mean and distributions of path loss signals that are treated as a random variable can be used to model the physical-layer wireless channel model. Authors in [126], have employed EC to estimate the delayoutage probability with log-normal fading distribution. In this work, the system performance of the CDMA networks has been investigated in detail. Network traffic has been modelled as a stochastic process, and then, extensive simulations have been performed to show the impact of network traffic on the achievable EC of the system with log-normal fading conditions. With this framework, the system capacity and traffic demands are predicted. Also, this EC-based anlsyis leads to the conclusion that, traffic correlation is good when the system load level is the same.

5) Weibull: This fading channel has been used in wireless communications with its implementation in indoor and outdoor environment. The EC model in wireless networks with Weibull fading channel has been discussed in [127]. To support realtime applications, an independent but not identical Weibull fading channel has been used to find and test the effective rate while considering the concept of EC. High SNR and low SNR-based closed-form asymptotic analysis has also been performed to find the optimal effective rate under other fading conditions as well. This work shows that, more stringent delay requirements minimizes the effective rate. Another very interesting conclusion from this work is that, the effective rate is prone to the severity of fading channels.

6) Generalized Fading Models: Compared to other fading models such as Rayleigh and Rician, generalized fading models provide a general framework with the combination of one or more fading model [233]. In general, EC concept can be used to analyze the performance of generalized channel fading models with various adaptive transmission policies under different fading and transmission constraints. Below is the description of different generalized fading conditions with EC metric analysis in different wireless networks:

Under the generalized fading model, $\alpha-\mu$ fading model uses $\alpha-\mu$ distribution. As compared to stochastic fading models, $\alpha-\mu$ distribution provides more generality to analyse the fading environment [128]. In [129], authors have used the EC model in underlay $\mathrm{CRNs}^{3}$ with $\alpha-\mu$ fading model. In this study, an in-depth performance analysis based on symbol error probability using EC concept has been performed with $\alpha-\mu$ fading conditions. In this work, the EC model has been employed to understand the peak interference power to noise

\footnotetext{
${ }^{3}$ for details on underlay CRNs, see Section VI.
}

ratio. The results suggest that when the delay becomes more stringent, the peak interference power to noise ratio becomes smaller. However, the peak interference power to noise ratio increases with the increase in EC. This analysis is useful for understanding the performance of cognitive cooperative networks over $\alpha-\mu$ fading channels.

How shadowing affects the performance in wireless networks, can also be investigated with the help of $\kappa-\mu$ fading conditions. $\kappa-\mu$ fading model provides a more general model by covering Gamma shadowing, one-sided Guassian, Nakagami-m, Rayleigh, and Rician fading. Authors in [130], [131], have discussed $\kappa-\mu$ fading model and analyzed the system gain through the concept of EC. Analytical expressions for MISO systems with the EC model under $\kappa-\mu$ fading conditions have been provided. MGF approach is employed to investigate the achievable EC of MISO systems. This work provides a useful insight into the MISO systems, by investigating the asymptotic analysis of the EC at high SNR. Without the concept of EC, these insights are not possible with any other model.

EC-based QoS analysis has also been performed with $\kappa$ fading channels to test the performance of shadowing and multipath propagations and their impact on received signal quality. Impact of generalized $\kappa$ fading conditions in cooperative communications has been analyzed with EC concept in [52]. Under the influence of generalized $\kappa$ fading conditions, it has been estimated that, increasing the number of relays can also maximize the performance of a system with delay constraints.

\section{B. Futuristic Models}

Some futuristic models such as matrix-exponential distribution (MED) and broadcast fading models have also been investigated with EC model in more detail. With the help of futuristic models, some aspects of wireless communications such as retransmission and compression schemes have been investigated with the EC model. Below is the description of some versions of futuristic fading models that have been investigated with EC.

1) MED: Different versions of retransmission schemes such as persistent and truncated ARQ and HARQ have been investigated with MED-based channel model in [226]. An ECbased analytical expression has been provided for different retransmission schemes ${ }^{4}$ while considering MED-based fading channel. With the help of this fading condition and the EC model, impact of diversity (due to MIMO antennas) on the system has been investigated in detail. This work also provides the EC expression for the persistent truncated, and networked HARQ, that was not addressed before. Under the MED-based fading channel and using the EC model, the target delay with delay violation probability has been estimated. This can lead to obtaining the important results regarding the general effective channel functions and transition probability. Another important take-away from this study is that, the diversity of the MIMO under MED fading channels, reduces the sensitivity of EC with respect to different delay exponents.

\footnotetext{
${ }^{4}$ for details on retransmission scheme, see Section VIII.
} 
2) Broadcast Fading Models: Fading in broadcast or multicast channels can also be investigated with the help of the EC model [228]. The EC concept with broadcast fading channels in mobile networks has been investigated in [227]. In broadcast channel, different fading states across the receiver has been taken into account while estimating the performance of a system with EC. A trade-off has been developed among delay constraints, QoS guarantee, throughput, and reliability. In this study, reliability has also been taken into account to estimate the overall packet loss.

3) Model for Unknown Fading Conditions: In some cases, there are scenarios, where statistics of a fading is unknown. For such links where fading statistics are unknown, authors in [229] have proposed a scheme where channels with unknown statistics can be modelled using the EC model. This scheme also takes into consideration an optimal power allocation and link selection scheme with EC. This introduces more flexibility in a system, as proposed approach has the capability to converge in any fading distributions depending upon the transmission scenarios and fading.

\section{Dimension-Based Models}

Dimension-based models are also called multi-ray fading models. As compared to other fading models, these models calculate path-loss along all possible paths or depending upon diverse fading conditions. The existing work considering dimension-based fading models with the EC model can be classified into two-dimensional (2D) and multi-dimensional fading models.

1) $2 D$ Fading Model: Achievable EC of wireless networks under 2D fading channel has been investigated in [160]. In this study, a 2D-based Markov model has been used to investigate the fluctuating channel conditions QoS at link-layer has been analyzed with EC. For this purpose, arrival rate/source rate is find with EC and when the arrival rate is fixed, the delay experienced by the arriving packet is estimated. Proposed scheme has been tested extensively with the EC model and compared with other schemes.

2) Multidimensional Fading Models: As compared to [160], in which 2D-based Markove process has been used to model the fading conditions, in [219] authors have employed a multi-dimensional-based Markov process to model fading conditions and then used the EC model to investigate the delay outage probability. In this study, a cross-layer resource management approach has been used with the EC model to investigate arrival-rate, queueing behavior, in multidimensional fading channels. Extensive simulation shows that proposed scheme achieves higher throughput while guaranteeing the required delay for QoS-aware applications.

\section{Supplementary Models}

Supplementary fading models have also been analysed with the EC model. Supplementary models are not the distinct class of fading models, but they are usually introduced to address certain limitations of existing fading channels. Supplementary fading models are actually based on existing fading models. Below is the description of the some supplementary fading models with EC theory, that has been proposed to address some special features or aspects in existing fading channels/models:

1) Turbulence Model: In advanced wireless communications systems, such as optical wireless communications (OWC), impact of turbulence fading has also been investigated with the EC model [220]. Following works [220][223] consider the turbulence fading with their achievable EC in OWC . In these studies turbulence fading conditions have only been analyzed for the OWC with different poweradaptation schemes. The closed-form expression of the EC with turbulence fading has also been derived and validated through extensive simulations.

2) Gamma Fading: As compared to turbulence fading, Gamma-Gamma turbulence fading has also been discussed in OWC in [224]. With the help of this fading distributions, the independent and joint power adaptation in OWC with their achievable EC have been investigated in detail. From this study, it has been clear that if the fading is minimized and a delay constraint is loose, then the performance gap between independent and joint power adaptation is minimal. Authors in [225], have utilized Gamma distribution for modelling wireless channel in CRNs with EC. Optimal power allocation with power/interference-power has been analyzed with statistical QoS provisioning. Proposed scheme has been extensively tested with its achievable EC through simulations and shows improved performance as compared to other state-of-the-art DSA techniques.

3) Temporal Fading: Supplementary fading models with temporal distributions have been investigated in smart grid environment [47]. In this study, the performance of wireless communications systems in smart grid environment has been investigated with the EC model. Fading conditions have been modeled using a temporal fading model. This EC-based delay analysis of smart grid's communications architecture shows adaptability of this architecture for different smart grid's applications.

\section{E. Summary and Insights}

Complexity of the EC model increases as the fading conditions become more and more complex. As compared to the ergodic and Shannon capacity, the closed form expression for EC by taking into consideration the different fading conditions is relatively more complex and difficult to obtain. This is a serious challenge for considering the EC over other capacities for testing the performance of any systems. EC has been extensively used with the i.i.d Rayleigh fading conditions, however, the accuracy of the EC model with non i.i.d fading conditions invites the future researchers to investigate further.

The EC model helps in understanding QoS-requirements with varying service rate under different fading conditions. Changes in EC according to different fading conditions provides a strong mathemtiacal tool for observing the effects of fading conditions on the delay performance of the considered fading channel. Through these changes, optimal resource allocation schemes, scheduling algorithms, and network designs can also be proposed and investigated in more detail. 


\section{EFFeCtive CAPacity Measures in DiffERENT NETWORKS}

With the help of the EC model, the performance of different wireless networks under different delay requirements can be investigated by considering various wireless designs and architectures. Achievable EC of CRNs, wireless sensor networks, relay-networks, and mesh networks have been analysed extensively in the literature. In this section, we have broadly discussed these networks with their different design and architectural aspects with the EC model.

\section{A. Cognitive Radio Networks (CRNs)}

Current spectrum crunch can be avoided with the help of cognitive radio networks (CRNs). CRNs employ dynamic spectrum access (DSA) approach and dynamically assign spectrum resources while avoiding the interference to primary users [241]. Cognitive users that use licensed spectrum band are termed as the secondary users (SUs), while users of licensed spectrum band are named as the primary users (PUs). In CRNs, SUs utilize the idle portion of licensed spectrum band while keeping minimum interference to PUs. Idle portions of licensed band are termed as white spaces [249], [250].

Various QoS-aware applications in CRNs can well be tested with the help of EC metric [172]. Source and service rate characterization is an important feature of the EC model. This feature has been well-exploited in CRNs. With achievable EC in CRNs, various techniques such as efficient spectrum sensing, resource allocation, modulation, spectrum access, and interference constraints can be analyzed.

Achievable EC in CRNs has been studied from the perspective of white space utilization. According to the utilization of white space, CRNs can be categorized into underlay, overlay, interweave, and hybrid CRNs [251], [110], [225]. Figure 3 shows the classification of existing work on EC-based CRNs into underlay, overlay, interweave, and hybrid CRNs.

Table III shows various gains with their corresponding study in different categories of CRNs with the EC model. Impact of PUs activity patterns in different classes of CRNs has also been presented. Below is the description of different classes of CRNs with the concept of EC.

1) Underlay CRNs: This white space utilization pattern is also named as gray-space utilization in CRNs. In this scheme, SUs can transmit simultaneously with PUs, while keeping the interference to PUs within an acceptable range. For this purpose, SUs have to use low-power cognitive devices with much limited range compared to other classes of CRNs. Spectrum sensing, modulation, and optimal resource allocation under different fading conditions in underlay CRNs have been well-investigated with the EC model. Underlay CRNs with their achievable EC with different spectrum sensing approaches have been discussed in [143], [145], [148], [234][236]. Most of the work on spectrum sensing with the EC model considers energy-detection-based spectrum sensing approach with predictable and known parameters. However, other spectrum sensing approaches, such as QoS-aware, cooperative, and cyclostationary-based spectrum sensing have not been explored while taking into consideration the EC model.
This gray-space utilization pattern of CRNs with the EC concept has also been used to develop various optimal resource allocation schemes with stringent-delay requirements [102], [110], [116], [124], [137], [153], [172], [237]. Among resource allocation strategy, problem of power allocation has been formulated and then solved by applying different optimization scenarios. However, radio resource allocation schemes (spectrum assignments) with the EC model for underlay CRNs have not been studied in detailed.

Statistical QoS-provisioning in underlay CRNs with the EC concept has also been studied in CR-based cooperative communications with single and multiple relays [142], [158], [195]. In these work, buffer-aided relaying strategy has been employed. The EC model in these underlay CRNs has also been used with varying fading conditions, such as generalized fading models, stochastic fading model, and Nakagami$m$ fading channel [115], [128], [129]. Adaptive modulation techniques have also been analyzed using the EC concept in underlay CRNs [118], [120], [203]. In these modulation schemes, average power consumption has been monitored with the provision of statistical QoS. Average and available rate have also been analysed while keeping the interference to PUs within minimum range.

2) Overlay CRNs: In overlay CRNs, SUs can exploit licensed spectral resources either cooperatively or noncooperatively, with or without the presence of PUs. SUs can simultaneously transmit with PUs by adjusting their transmission parameters to keep the interference to PUs at a minimal and acceptable limit. As compared to underlay CRNs, in overlay CRNs, PUs can acknowledge the presenece of SUs at a licensed spectrum band (in case of cooperative communications) [250].

The EC model with overlay CRNs has been used while analysing different aspects of overlay CRNs, such as spectrum sensing, resource allocation, and channel accessing methods. EC concept with overlay CRNs has been used with different spectrum sensing approaches such as discussed in [157], [198], [240], [241]. In these work, efficient spectrum sensing approaches based on required delay-outage probability has been investigated with the achievable EC while keeping the interference to PUs at a minimal range are obtained. Optimal resource allocation schemes in overlay CRNs [37], [44], [58], [60], [103], [107], [177], [181], [202], [225], [242]-[245] with interference constraints [155], [238] has also been investigated with EC. In addition to these resource allocation schemes, FD communications behavior for overlay CRNs have also been analyzed with the EC model. Antenna diversity with multiple input and multiple output (MISO) antennas in overlay CRNs has been analysed with the EC concept [239]. In this study, channel estimation errors have been evaluated with their impact on the performance of overlay CRNs with MISO antennas.

3) Interweave CRNs: When there is no PUs activity in a licensed band, white space utilization by SUs in this case is termed as an interweave white space utilization or interweave CRNs [250], [252]. In interweave CRNs, SUs can only utilize licensed spectrum resources when PUs is idle.

Most of the heterogeneous real-time applications are sup- 
TABLE III

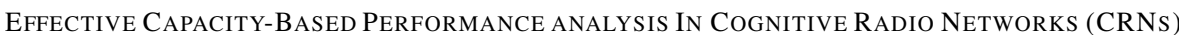

\begin{tabular}{|c|c|c|c|c|c|}
\hline $\begin{array}{l}\text { White Space } \\
\text { Paradigm }\end{array}$ & Study & $\begin{array}{l}\text { PUs } \\
\text { Activ- } \\
\text { ity }\end{array}$ & Factors evaluated with EC metric & $\begin{array}{l}\text { Fading Channel } \\
\text { Model }\end{array}$ & Area \\
\hline \multirow{23}{*}{ Underlay CRNs } & [143] & & Channel Errors & Rayleigh & Spectrum Sharing with Imperfect CSI \\
\hline & [145] & $\checkmark$ & QoS with Interference Limitations & Rayleigh & Multiple Channel Spectrum Sharing \\
\hline & [148] & $\checkmark$ & Interference Limitations & Rayleigh & \multirow[t]{3}{*}{ Spectrum Sensing In CRNs } \\
\hline & {$[235]$} & $\checkmark$ & Channel Gain & Rayleigh & \\
\hline & [236] & & Analysis of Outdated CSI & Not Defined & \\
\hline & [128] & & General Fading & $\alpha-\mu$ & \multirow{3}{*}{ CRNs with Various Fading Conditions } \\
\hline & [129] & & General Fading & $\alpha-\mu$ & \\
\hline & [115] & & Different QoS Applications & Nakagami- $m$ & \\
\hline & [234] & $\checkmark$ & Optimal Power Allocation & Rayleigh & \multirow[t]{8}{*}{ Resource Allocation } \\
\hline & [137] & & $\begin{array}{l}\text { Trade-off Between SUs and PUs per- } \\
\text { formance }\end{array}$ & Rayleigh & \\
\hline & [237] & $\checkmark$ & Interference Power Constraint & Gamma Fading & \\
\hline & [153] & & Power Allocation with Imperfect CSI & Rayleigh & \\
\hline & [102] & $\checkmark$ & Power Allocation with Imperfect CSI & Rayleigh & \\
\hline & [110] & & Securing the CRNs & Nakagami- $m$ & \\
\hline & [172] & & Optimal Power Allocation & Rayleigh & \\
\hline & [116] & $\checkmark$ & Optimal Power Allocation & Nakagami- $m$ & \\
\hline & [124] & & Optimal Power Allocation & Nakagami- $M$ & CR-Based MAC \\
\hline & [118] & $\checkmark$ & $\begin{array}{l}\text { Rate and Power Estimation with } \\
\text { MQAM }\end{array}$ & Nakagami- $m$ & \multirow[t]{2}{*}{ Modulation In CRNs } \\
\hline & [120] & $\checkmark$ & Power Estimation with MQAM & Nakagami- $m$ & \\
\hline & [203] & & SUs Throughput & Rayleigh & FDMA-Based CRNs \\
\hline & [142] & & SUs arrival rate & Rayleigh & \multirow[t]{3}{*}{ CR-Relay Networks } \\
\hline & [158] & $\checkmark$ & Interference and Delay Constraints & Rayleigh & \\
\hline & [195] & & SUs Capacity & Rayleigh & \\
\hline \multirow[t]{21}{*}{ Overlay CRNs } & [157] & $\checkmark$ & Power Level based on PUs & Rayleigh & \multirow[t]{2}{*}{ Spectrum Sensing In CRNs } \\
\hline & {$[198]$} & $\checkmark$ & SUs Arrival Rate & Rayleigh & \\
\hline & [155] & & Performance Limitations of SUs & Rayleigh & \multirow[t]{2}{*}{ Interference Limitations In CRNs } \\
\hline & [238] & $\checkmark$ & SUs Capacity Analysis & $\begin{array}{l}\text { Asymmetric Fad- } \\
\text { ing }\end{array}$ & \\
\hline & [239] & & Channel Estimation Error & Nakagami- $m$ & CRNs with MISO Systems \\
\hline & [240] & $\checkmark$ & Two phase channel Access Method & Not Defined & \multirow[t]{2}{*}{ Channel Access In CRNs } \\
\hline & [241] & $\checkmark$ & Hopping Based Channel Access & Not Defined & \\
\hline & [225] & $\checkmark$ & Optimal Power Allocation & Gamma Fading & \multirow[t]{14}{*}{ Resource Allocation } \\
\hline & [103] & & SUs Arrival Rate & Nakagami- $m$ & \\
\hline & [242] & $\checkmark$ & Optimal Power Allocation & Nakagami $m$ & \\
\hline & [58] & & Optimal Resource Allocation & Rayleigh & \\
\hline & [60] & $\checkmark$ & Optimal Power Allocation & Nakagami- $m$ & \\
\hline & [107] & $\checkmark$ & Full-Duplex Operation & Nakagami- $m$ & \\
\hline & [243] & & Optimal Power Allocation & Rayleigh & \\
\hline & [244] & & Power Consumption & Rayleigh & \\
\hline & [245] & $\checkmark$ & Optimal Resource Allocation & Not Defined & \\
\hline & [177] & $\checkmark$ & Optimal Time Slot Allocation & Rayleigh & \\
\hline & [181] & & Optimal Channel and Power Allocation & Rayleigh & \\
\hline & [44] & & Cross-tier Interference & Rayleigh & \\
\hline & [37] & & Cross-tier Interference & Rayleigh & \\
\hline & [202] & & QoS-Based Power Allocation & Rayleigh & \\
\hline \multirow[t]{9}{*}{ Interweave CRNs } & [15] & $\checkmark$ & Optimal Sensing Time Achieved & Nakagami- $m$ & Spectrum Sensing In CRNs \\
\hline & [140] & $\checkmark$ & Interference Power Limitations & Rayleigh & Interference Constraints \\
\hline & [92] & $\checkmark$ & QoS in Cognitive M2M & Rayleigh & Cognitive M2M Communications \\
\hline & [246] & $\checkmark$ & Voice Capacity & Not Defined & Voice Capacity in CRNs \\
\hline & [247] & $\checkmark$ & Voice Capacity & Rayleigh & Wireless Multimedia CRNs \\
\hline & [248] & $\checkmark$ & Delay Bound & Not Defined & \multirow[t]{2}{*}{ Resource Allocation } \\
\hline & [199] & $\checkmark$ & SUs Capacity & Rayleigh & \\
\hline & [98] & $\checkmark$ & Optimal SUs Transmission Rate & Nakagami- $m$ & CRNs performance Without CSI \\
\hline & {$[252]$} & & SUs performance with Imperfect CSI & Rayleigh & GSC In CRNs \\
\hline \multirow{2}{*}{ Hybrid CRNs } & [134] & $\checkmark$ & Complexity and Cost & Rayleigh & PUs Spectrum Sensing Feedback \\
\hline & [141] & $\checkmark$ & SUs performance Quantified & Rayleigh & Spectrum Sensing In CRNs \\
\hline
\end{tabular}




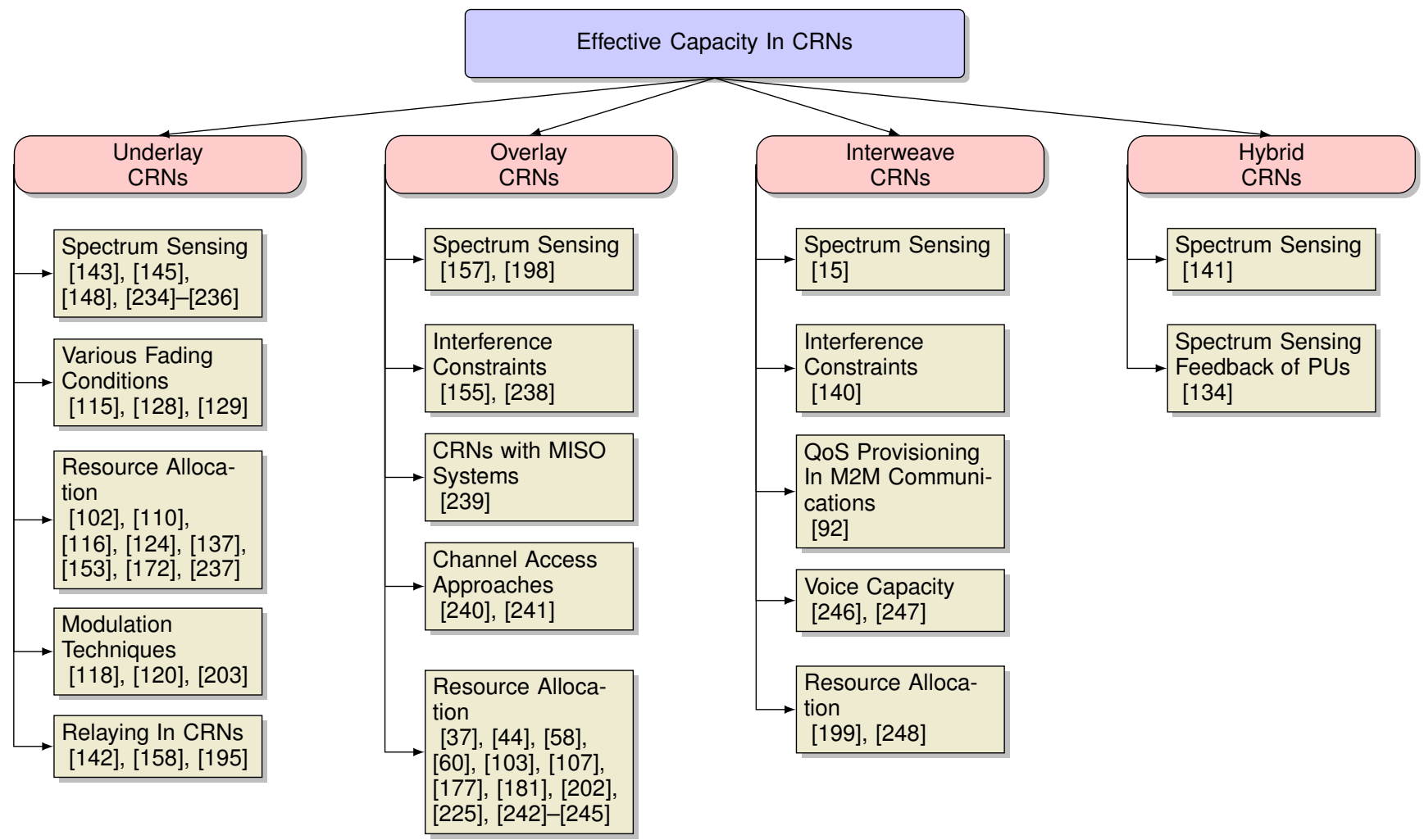

Fig. 3. Effective capacity in CRNs can be studied while employing various white space paradigm such as overlay, underlay, Interweave, and hybrid CRNs.

ported by interweave CRNs. EC concept in interweave CRNs has also been used to analyse the statistical QoS provisioning with delay constraints for different real-time, QoS-aware, and bandwidth hungry applications such as multimedia applications [92], [246], [247]. Energy-detection based spectrum sensing [15], interference constraints [140], and resource allocation scheme [199], [248], have also been analysed with achievable $\mathrm{EC}$ in interweave CRNs. Impact of PUs activity has also been studied with the EC concept. However, different PUs activity patterns, such as low, high, and intermittent PUs activity with their impact on interweave CRNs has not been investigated while considering the EC model. This opens the new vistas for future research directions.

4) Hybrid CRNs: In most cases, SUs can exploit idle spectrum resources by adopting a hybrid approach of white space utilization (combination of above two or three) [250]. By applying a hybrid approach, limitations of above mentioned approaches can be overcomed. However, this approach introduces some type of complexity in managing different factors such as PUs activity and spectrum mobility. Authors in [134], [141] have modelled the queueing-delay with the help of EC in hybrid CRNs with spectrum sensing paradigm. In this study, feedback from PUs for interference has also been analyzed. This feedback is then taken into account for designing an optimal scheduling approach. Queue length information with PUs feedback is used for guaranteeing the statistical QoS with efficient spectrum sensing approach. A tradeoff between the SUs achievable EC and the PUs success rate has been characterized. From this tradeoff, a three level power allocation scheme is then developed to enhance the
QoS-aware performance of the SUs.

\section{B. Cooperative Networks}

Due to the advancement in relaying protocols, cooperative networks have gained much attention. In cooperative networks, a source node can communicate with a destination node with some intermediate nodes/relays. Statistical QoS-provisioning has been studied by characterizing the traffic not only on a source node but also at a relay node [253]. Table IV shows the recent work based on the EC model in cooperative networks. Existing work on EC-based QoS estimation in cooperative networks has been classified based on the relay selection approaches.

In traditional cooperative networks, relaying is performed either in regenerative or non-regenerative way. When a regenerative approach is considered, a decode-and-forward (DF) relaying method is used, while non-regenerative approach employs the amplify-and-forward (AF) scheme in selecting a relay node. Advance wireless networks also consider other relaying approach such as QoS-aware relaying, buffer-aided relaying, and detect-and-forward relaying approach. Achievable EC of cooperative networks with different relaying schemes has been surveyed below:

1) Amplify-and-Forward Relaying: Achievable EC for nonregenerative approach of relaying named as amplify-andforward (AF) relaying scheme has been discussed in [260]. EC of AF relaying scheme has been studied extensively in existing work as compared to other relaying scheme such as DF [260]. DF relaying performs some type of processing before sending the packets. The concept of achievable EC in AF 
TABLE IV

EFFective CAPACITY-BASEd Performance Testing In CoOPERATIVE Networks

\begin{tabular}{|c|c|c|c|}
\hline Relaying Method & Architecture or Design Involved & Study & Factors evaluated with EC metric \\
\hline \multirow[t]{7}{*}{ Amplify-and-Forward } & Multi-User Cooperative Network & [93] & Optimal Resource Allocation \\
\hline & Multi-Hop Network Design & [94] & $\begin{array}{l}\text { EC performance Analysis with Channel State Infor- } \\
\text { mation }\end{array}$ \\
\hline & Multi-Relay Networks & [144] & Optimal Resource Allocation \\
\hline & Two-Way Relay Networks & {$[254]$} & $\begin{array}{l}\text { Analysis of Various Gains of Two-Way Relay Net- } \\
\text { works }\end{array}$ \\
\hline & Full-Duplex OWC Network & {$[221]$} & $\begin{array}{l}\text { Optimal Resource Allocation with Throughput Max- } \\
\text { imization }\end{array}$ \\
\hline & Single Relay Network & [167] & Cross-Layer Power Allocation \\
\hline & Virtualized Relay Networks & {$[112]$} & Optimal Power Allocation \\
\hline \multirow[t]{4}{*}{ Decode-And-Forward } & \multirow[t]{2}{*}{ Multi-Relay Networks } & {$[146]$} & Relaying Scheme with Modulation \\
\hline & & {$[150]$} & EC Analysis With Four Retransmissions Schemes \\
\hline & Full-Duplex Relay Networks & {$[62]$} & Optima Resource Allocation \\
\hline & Three-Mode Relay Networks & {$[255]$} & Adaptive Relaying \\
\hline \multirow[t]{8}{*}{ Buffer-Aided } & \multirow[t]{2}{*}{ Three Node Relay Networks } & {$[135]$} & EC-Analysis with Buffer Aided Relaying \\
\hline & & {$[174]$} & EC-Analysis with Buffer Aided Relaying \\
\hline & Full-Duplex Relay Networks & [31] & EC-Analysis with Buffer Aided Relaying \\
\hline & \multirow[t]{3}{*}{ Two-Hop Networks } & {$[256]$} & Resource Allocation \\
\hline & & [257] & Concurrent Relay Selection Has been proposed \\
\hline & & [151] & $\begin{array}{l}\text { Buffer Constraints and Throughput Has been ex- } \\
\text { plored }\end{array}$ \\
\hline & \multirow{2}{*}{$\begin{array}{l}\text { Diamond Relay Networks with two } \\
\text { Relays }\end{array}$} & {$[258]$} & Performance analysis with New Selection Policy \\
\hline & & [259] & Performance analysis with New Selection Policy \\
\hline \multirow[t]{6}{*}{ QoS-Based } & Multi-Relay Networks & {$[104]$} & Optimal Resource Allocation \\
\hline & Heterogeneous Relay Networks & [190] & Optimal Resource Allocation \\
\hline & Two-way Relay Network & [196] & Optimal Cross-Layer Resource Allocation \\
\hline & Mobile Multi-Hop Relay Networks & [45] & Cross-Layer Simulation Platform \\
\hline & \multirow[t]{2}{*}{ Two-Hop Networks } & [207] & TDMA and OFDMA-Based Resource Allocation \\
\hline & & [208] & TDMA and OFDMA-Based Resource Allocation \\
\hline \multirow[t]{3}{*}{ Frequency-Based } & \multirow[t]{2}{*}{ OFDMA-Based Relay Networks } & [194] & Optimal Resource Allocation \\
\hline & & [197] & Optimal Resource Allocation \\
\hline & CR-Based Relay Networks & [198] & $\begin{array}{l}\text { Cross-Layer Resource Allocation with Imperfect } \\
\text { Spectrum Sensing }\end{array}$ \\
\hline \multirow{2}{*}{$\begin{array}{l}\text { Amplify-And-Forward, Decode- } \\
\text { And-Forward }\end{array}$} & Full-Duplex Relay Networks & {$[32]$} & Optimal Resource Allocation \\
\hline & Two-Hop Relay Networks & [209] & Cross-Layer Resource Allocation \\
\hline Detect-And-Forward & Multi-Relay Networks & {$[105]$} & Optimal Resource Allocation \\
\hline
\end{tabular}

relaying has also been exploited to evaluate the performance of FD cooperative communications [221], multi-user cooperative networks [93], multi-hop networks [94], [144], two-way relaying [254], single-relaying [167], and virtualized relaying [112]. AF relaying protocol with EC concept in most cases considers optimal power allocation schemes to achieve energy conservation in energy-scarce networks. However, other issues such as radio resource allocation in CR-based relay networks has not been investigated with the EC model. This invites the future researchers to investigate further in this domain.

2) Decode-and-Forward Relaying: Decode-and-forward (DF) relaying is a regenerative relaying approach that regenerates or decode the packets first before transmission to another node in cooperative networks. Investigating the achievable EC of DF relaying scheme is more complex as compared to $\mathrm{AF}$ relaying. $\mathrm{DF}$ relaying is quite similar to "processing base station", hence characterization of source traffic based on delay constraints on processing base station or relay node adds more complexity in the system. This complexity has been minimized by adopting an advance DF-base relaying network architectures such as FD-relaying [62], three-mode relaying [255], and multi-relaying [146], [150].

3) Buffer-Aided Relaying: Buffer-aided relaying is a subtype of $\mathrm{AF}$ and $\mathrm{DF}$ relaying. Buffer-aided relaying can be performed by adopting any relaying protocol (AF or DF) with additional buffers [135], [229]. Cooperative networks with buffer-aided relaying can support variable source rate by adjustable buffers at source and the relay nodes and their performance has been tested with the EC model. EC concept in buffer-aided relaying with advance communications designs/architectures such as three-node relay networks [135], [174], FD-relay networks [31], two-hop networks [151], [256], [257], and diamond-relay networks [258], [259] has also been used to investigate the performance of these advance networks with under delay-violation probability constraints.

4) QoS-Based Relaying: QoS-based relaying can be performed with the help of regenerative, i.e., DF or nonregenerative, i.e., AF approach of relaying. This relaying approach has specially been introduced to incorporate the demand of stringent delay requirements at the relay nodes. EC concept has also been investigated with this relaying approach by ensuring statistical QoS provisioning. This relaying approach with EC concept has further been used to analyze multi-relays [104], heterogeneous relays [190], twoway relaying [196], mobile multi-hop relaying [45], and two-hop networks [207], [208]. QoS-based relaying shows improved performance as compared to other relaying schemes when considering multimedia applications with stringent delay 
requirements. These studies provide important insights, such as, the EC can be improved by properly adjusting the location of the relays. Furthermore, when the relay networks are investigated with the FDMA and TDMA approaches with their achievable EC, it has turned out that FDMA approache outperforms TDMA.

5) Frequency-Based Relaying: Relaying can also be performed by considering frequency domain of a wireless communication paradigm. By considering frequency as a decision factor for selecting the relay, issue of spectrum scarcity can also be addressed [194], [261]. Multimedia applications with the help of the EC model has also been analyzed in frequencyaware relaying. OFDMA-based relay networks [194], [197] and CR-relay networks [198] consider frequency-based relay selection approach and employ the EC model to test the performance of a system.

6) Amplify-and-Forward and Decode-and-Forward Relaying: Hybrid relaying approach comprising of AF and DF relaying approach has also been used in many advance wireless networks. With a hybrid approach, limitations of AF and DF relaying schemes can be addressed while supporting QoSaware applications with specific delay requirements [209]. This approach of relaying in conjunction with FD-relaying [32] and two-hop relaying [209] has also been analysed with EC concept with the required delay-violation probability. Achievable EC of both relaying schemes has been compared with the direct transmission while taking into consideration the stringent delay requirements. This analysis shows the superior performance of both relaying schemes as compared to the direct transmission when delay requirements are stringent.

7) Detect-and Forward Relaying: In comparison to AF and DF relaying, detect-and-forward (DeF) relaying has also been analyzed with the EC model. In [105], DeF relaying approach takes into consideration the achievable EC in multi-relay networks. First, the best channel is detected on that basis the relay is selected. With the increase in QoS-requirements with stringent delay requirements, number of relays are increased accordingly. In this way, this scheme adds more flexibility in a system to achieve certain QoS requirements.

\section{Optical Networks}

Concept of EC has also been used in OWC [223]. OWC utilizes ultraviolet, visible, and infrared light as a wireless medium to transmit signal [222], [262]. Visibl light communications ${ }^{5}$ is also a type of optical communications, that operates in a visible spectrum band (390-750nm). Extensive work has been done on optical wireless communications with respect to EC concept. Authors in [263], have analysed the performance of MIMO antennas in optical communications with the help of the EC model. As compared to traditional wireless networks, optical communications employ turbulence fading channels to accommodate fading conditions of optical environment. In this study, Gamma-Gamma turbulence fading condition with MIMO antennas to support multiple users have been analysed with the EC metric with stringent delay requirements.

\footnotetext{
${ }^{5}$ for more details on achievable EC in visible light communications, see Section III.
}

An innovative and highly robust optical network framework named as petaweb has been proposed in [264], [265]. In this study, an in-depth analysis of optical networks with the EC model has also been provided with the consideration of IP networks. In this work, periodic fluctuations in a channel has been controlled through regulating a traffic flow.

\section{WLAN}

Wireless local area network (WLAN) provides a connectivity of two or more computers or devices over a limited area. WLAN follows the standard 802.11, and can be connected to Internet through a gateway [136], [266], [267]. EC concept in WLAN has been used to investigate the performance of WLAN [64], [203], [268] for multimedia applications. ECbased QoS analysis in WiFi networks has been discussed in [57]. In this study, WiFi offloading with heterogeneous architecture has been explored with statistical QoS provisioning. An optimal resource allocation scheme (power allocation) has been developed using the EC model. For efficient estimation of available bandwidth in WLAN, WBest (a bandwidth estimation tool based on the EC model in WLAN) has been proposed in [76]. WBest operates with an algorithm that comprises of two steps. In the first step, achievable EC is estimated with the help a packet-pair approach, while the second step provides the throughput analysis. This tool has been tested with many multimedia applications that demand higher bandwidth and stringent delay requirement for their transmission.

WLAN-based single-hop adhoc networks with the EC model has been discussed in [269]. In this work, call admission control has been investigated with statistical delay guarantee. An efficient resource allocation algorithm has also been developed based on the EC model. Statistical QoS-provisioning in WLAN by considering the EC model has also been studied in [270], [271]. In this study, 802.11 based mobile station is considered as a server. This server is then modeled as the Markovian bursty server. The known results or activity patterns from this server is then used to derive EC for delay-sensitive and QoS-aware applications. Proposed scheme has been tested through extensive simulations to validate the operation of the work.

\section{E. Wireless Sensor Networks}

Advances in micro-electro-mechanical systems (MEMS) technology has resulted into the wireless sensor networks (WSNs). WSNs now have found their applications ranging from civil, medical, to military [272]-[275]. Limited battery life of tiny sensor nodes compel the researchers to come out with energy-conservation approaches while maximizing network life time and throughput [276]. Broad concept of EC has also been used in WSNs for investigating delay and jitter. Performance of sensing operation and energy conservation approaches has been studied using the EC model in [43]. Authors in [74], have discussed a wireless link scheduling approach with achievable EC in WSNs. Proposed scheduling approach assigns time-slots to different users based on source/arrival rate and required delay constrained. Then EC-based link-layer model is used to analyse this proposed scheduling approach 
to support QoS-aware applications. Proposed scheme has been compared with traditional time-division multiple-access (TDMA) scheme and shows an improvement in throughput and energy-efficiency. Support of heterogeneous multimedia applications over cluster-based WSNs with the EC model has been studied in [204]. In this work, two-tier architecture for tiny sensing nodes has been proposed. In this two-tier architecture, sensing nodes are provided with one antenna while the base stations (BS) are equipped with multiple antennas. Low latency applications are then sensed and transmitted over this architecture. Performance of the network is then investigated using the EC model.

\section{F. Mobile Wireless Networks}

Mobility consideration in wireless networks demands efficient management of wireless resources that should be available to all the mobile users [121]. The main goal of any mobile network is to enhance the flexibility and to reduce the cost of a required architectural layout [212], [213]. Efficient resource management can be introduced in mobile networks and the performance of the network can also be investigated using the EC model. Authors in [123], have provided a QoS-driven resource allocation scheme for mobile wireless networks. In this work, the problem of power allocation and rate adaptation has been discussed with aim to maximize the throughput. This QoS-aware resource allocation has been developed by considering the EC model.

Cross-layer resource allocation with the EC model to analyze the behavior of QoS-driven applications in mobile wireless networks has also been discussed in [215]. In this cross-layer model, MIMO antenna diversity has been modeled using a finite state Markove chain process, while the QoSprovisioning at link-layer has been modeled using the EC concept. This scheme shows an efficient interaction between the physical-layer and upper-layers while satisfying a QoSguarantee. Mobility issue with the EC modeling has also been analyzed in mobile satellite networks [232]. In this scheme, a statistical QoS-based power allocation has been proposed while supporting a high quality satellite wireless link. In this approach, physical wireless channel has been modeled as a shadowed Rician model. The performance of a proposed scheme in supporting QoS-aware for delay-sensitive applications has been analyzed using the EC model. An optimal resource allocation scheme to maximize the $\mathrm{EC}$ has been developed. The achievable EC has been studied with respect to different propagation conditions, delay exponent, and elavation angle. This study further confirms that, the achievable EC highly depends on the elevation angle in the mobile satellite networks.

\section{G. Vehicular Adhoc Networks}

With the help of vehicular-adhoc Networks (VANETs), dream of intelligent transportation in future smart cities can be realized [277]. However, this requires an intelligent management of different vehicular resources [278], [279]. Different multimedia applications such as streaming videos in moving vehicles have also been investigated with EC concept [280].
In this research work, the packet-delivery ratio between the vehicle and road-side unit has been analyzed. The EC model is used to estimate the optimal distance between a vehicle and road-side unit and to investigate the transmission of QoSaware data with loose delay requirements. Provisioning of low latency in vehicular communications with EC perspective has been studied in [281]. The concept of latency violation probability (LVP) with optimal resource allocation such as power and spectrum has also been investigated. This EC-based latency analysis shows that the LVP can well be characterized with the notion of EC. By investigating the different latency requirements of safety-critical information, the sum rate of vehicle-to-infrastructure (V2I) and vehicle-to-vehicle (V2V) links has been optimized by optimally allocating the resources, i.e., power and spectrum.

\section{H. Mesh Networks}

To minimize the dependency on one or more than one nodes for relaying the information to the destination, mesh networks have been proposed. In these networks, participating nodes try to connect to as many as possible nodes for relaying their information to the destinations [48]. EC concept has also been utilized in this network topology with an aim to analyse the required delay and QoS. In [211], authors have proposed the wireless multi-hop mesh network to support delay-sensitive applications. To investigate this mesh-topology, the EC model has been used. At the physical-layer, Rayleigh fading with fluid traffic model has been used. Extensive simulations have been performed under different traffic flows to validate the effectiveness of a proposed scheme. This study reveals that, in the scenario of multi-hop mesh networks, the route selection (routing path) is independent from the QoS requirements and other channel conditions. Furthermore, EC-based analysis of this multi-hop network shows that, the delay performance between the intermediate nodes is less sensitive to the arrival rate as compared to the end-to-end delay analysis.

\section{Cellular Networks}

Performance of cellular networks can also be analysed with the help of the EC model while considering delay constraints. Study of cellular networks with the EC model has been classified into LTE, small cells, femto cells, and 5G networks, as has been shown in Figure 4.

1) Traditional Cellular Networks: Advances in telecommunication systems have resulted into the emergence of advance cellular networks such as LTE and 5G. In cellular networks, area for which the radio connectivity is to be provided is usually divided into cells with hexagonal or with some other shape according to terrain and land characterization [22]. With an increase in cellular users, demand for advance applications such as multimedia has also been increased. To guarantee statistical QoS provisioning in cellular networks, EC-based modeling of cellular radio has been performed in [168]. In this work, the achievable EC of D2D communications underlaying cellular networks has been investigated in detail. A constraint optimization problem that can maximize the cellular users EC has been formulated. This study reveals that as the arrival rate 


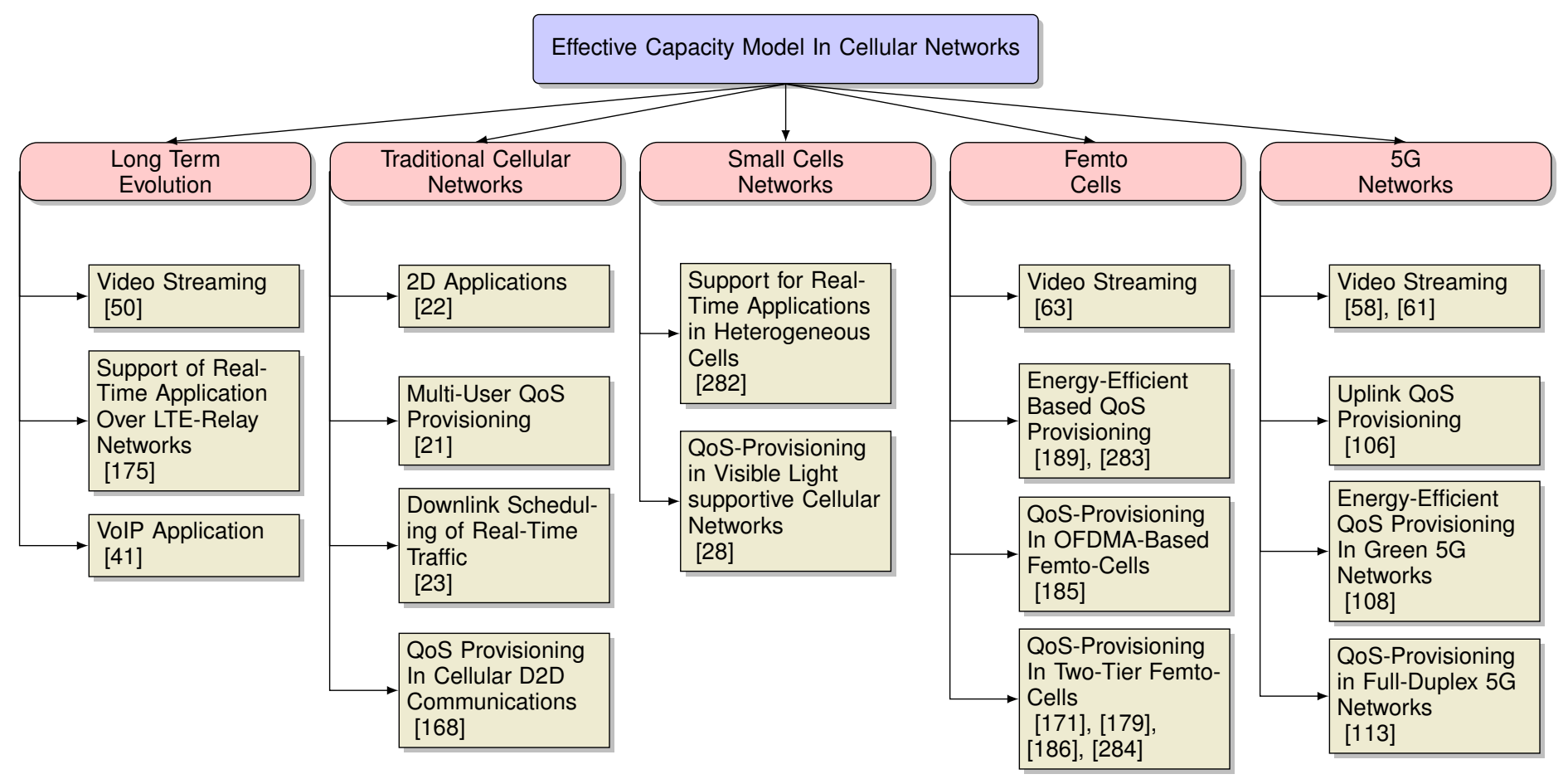

Fig. 4. Effective capacity measurements in cellular networks can be classified into traditional cellular networks, small Cells, Femto-cells, LTE, and 5G Networks.

of D2D pairs increases, the EC decreases and interference to the BS increases. This framework is then used to develop an adaptive power allocation scheme. Authors in [21], have used the EC model to investigate the optimal resource allocation scheme for downlink in cellular networks. In this study, multiuser statistical QoS-provisioning in downlink cellular networks has been taken into consideration. Problem of downlink resource allocation has been formulated with power and delay constraints into optimization problem and then solved using two-steps procedure. In this work, EC of downlink users with adaptive power allocation scheme has been compared with equal-length time division (TD) approach. The proposed scheme outperforms the equal-length TD with respect to the achievable EC of two users.

2) Femto Cells: To extend the coverage of existing cellular networks, concept of femto cells has been proposed in [63], [283], [285]. At the edge of cellular networks, where the coverage is unavailable or limited, femto-cell technology is usually used. It can accommodate approximately eight to ten users depending upon the infrastructure used. The performance of femto-cells have been studied with the EC model in [171], [179], [189]. In [185], downlink resource allocation for femto cells have been studied with the EC model. Statistical QoS provisioning has been ensured with a minimum energy consumption. In this OFDMA-based resource allocation scheme, channel and power allocation have also been analysed using the EC model for downlink femto cells. Authors in [186], have come out with an energy-efficient QoS-provisioning method based on the EC concept in uplink femto-cells. A two tierarchitecture consisting of macro-users and femto-cells has been proposed in this study. Interaction between two users for accessing random resources and supporting QoS-aware applications has been addressed with a Q-learning approach. The proposed scheme has been investigated with the EC model and shows improved performance in terms of convergence speed. Energy-efficiency with statistical QoS-provisioning in two-tier femtocells have also been analysed with the EC model in [284]. In this scheme, a price-based power control policy has been explored to mitigate inter-cell interference. Problem of power allocation has been formulated as a Nash equilibrium and is solved using a particle swarm optimization scheme. The EC-based delay and performance analysis helps to validate the effectiveness of the proposed scheme.

3) $5 G$ and Byond $5 G$ Networks: 5th generation (5G) is the next-generation wireless networks that promises higher bandwidth, lower-latency, ultra-reliability, and other features such as lower energy-consumption [58]. With higher data rates, $5 \mathrm{G}$ networks will have the flexibility to meet the needs of future implementation of Internet of Things (IoT) [286]. Higher data rates in $5 \mathrm{G}$ networks can be provided with the help of advance technologies such as FD communications, mmWave, and NOMA [61]. Achievable EC of 5G networks is used to investigate the performance of low-latency applications such as URLLC [106], [287]. Green 5G wireless mobile networks with the EC model has also been discussed in [108]. In this study, a statistical QoS-driven power allocation in 5G networks with SISO and MIMO antennas has been discussed. Concept of effective power efficiency (EPE) has been used in conjunction with the achievable EC. EPE for the SISO and MIMO antennas has been investigated through extensive simulations involving different delay bounds. 5G mobile networks with EC concept to analyse the performance of heterogeneous network resources has also been discussed in [113]. In this scheme, for the first time channel coupling has been studied 
using the EC model. In this study, to thoroughly understand the impact of channel coupling on network performance, three different case studies, i.e., FD communications, CRNs, and D2D communications, based on the EC model have been presented. In this EC-based diverse delay analysis of different technologies, the heterogeneous delay bounded proposed achitecture has been compared with the traditional homogeneous delay bound QoS schemes. The achievable EC of the proposed architecture outperforms the traditional homogeneous delay bound QoS schemes. Furthermore, different delay bounds of the BS depending on various enabling technologies of $5 \mathrm{G}$ has been chalked out.

4) Small Cells: To increase a cellular network capacity, flexibility, and resiliency, concept of small cells have been envisioned as the extension to long-term evolution (LTE). Small cells can reuse the existing licensed and unlicensed spectral resources, hence can improve the usage of spectral resources [28]. Small cells can include femto, macro, and micro-cells. EC concept has been used under the small cells umbrella. In [282], the installation of few small cells (from 1 to 3 ) in heterogeneous networks environment has been analyzed using the EC model. In this study, EC relief option related to analysing, planning, and management of small cells have been investigated in detail. EC analysis of this relief option shows that, properly deployed fewer small cells (1 to 3) can enhanced the network capacity three times. This deployment will also enhance the non-uniformity in traffic load.

5) LTE: 3rd generation partnership project (3GPP) has introduced the long-term evolution (LTE) that is based on existing GSM and UMTS standards [288], [289]. LTE with its new release called as 4 th generation $(4 \mathrm{G})$ can promise higher data rates and capacity for mobile users [50]. Performance of LTE networks has also been tested using the concept of EC metric [175]. Air-interface in LTE networks can support delay-sensitive and bandwidth hungry applications. Crosslayer scheduling scheme involving EC-based modeling of linklayer in LTE has been studied in [41]. In this downlink LTE networks, QoS-driven energy optimization has been analysed with the EC model. Extensive simulations of the proposed scheduling scheme shows $48 \%$ of reduction in total energyconsumption as compared to other traditional LTE frameworks.

\section{J. Summary and Insights}

In this section, different wireless networks such as CRNs, cooperative networks, optical networks, and cellular networks with their achievable EC are surveyed in detail. Maximization in EC in different networks are studied to understand the QoS provisioning for delay-sensitive applications. This will also be a corner stone to study the future networks with stringent latency requirements. Depending upon the white space utilization, CRNs can be categorized into underlay, overlay, and interweave CRNs. However, it is a challenging task to determine which technique is suitable for applications with stringent or loose delay constraints. The EC model can be a helpful tool to explore this issue further. In CRNs, another challenge is to find out the quantification between different techniques such as delay, reliability, spectrum sensing, PUs activity, and spectrum mobility. In this regard, using the EC metric can simplify the performance analysis of CRNs to understand the relation/trade-off between different techniques of spectrum sensing, PUs activity, delay, and others.

In cooperative networks, $70 \%$ of the delay and energydepletion is due to the relays and BS's. There exists a serious challenge to understand the performance of cooperative networks with delay-constraints. To address this challenge, EC provides a clean slate and is considered as an accurate and flexible tool to understand performance of cooperative networks.

\section{EFFECTIVE CAPACITY FOR FUll-DUPleX COMMUNICATIONS}

Full-duplex (FD) communication has gained much attention due to the provision of higher data rates [61]. QoSaware multimedia applications are often regarded as bandwidth hungry. Therefore, concept of FD communications has been introduced to support the ballooning demand of real-time and delay-sensitive applications. However, dream of simultaneous transmission and reception (FD communications) can only be realized while suppressing self-interference (SI).

Concept of link-layer channel model, i.e., EC for analysing the performance of FD communications has been extensively explored in existing literature. EC-based delay analysis with half-duplex communications design has been provided in detail in [146], [151], [254]. In comparison to half-duplex communications, FD design can theoretically double data rate. Potential advantages of FD communication can only be achieved with the implementation of proper self-interference suppression (SIS) approaches.

In this Section, we have considered the achievable EC of FD communication with different SIS approaches under delay-outage probability constraints. Self-interference in FD communications can be minimized with the help of either passive or active SIS approaches. In passive SIS approach, SI is mitigated with antenna and signal propagation approaches, such as antenna shielding, antenna separation, beamforming, and antenna polarization effects. In active SIS approach, radio frequency (RF) canceller and baseband canceller are utilized to achieve simultaneous transmission and reception. Table $\mathrm{V}$ shows a comparative view of different SIS approaches while highlighting the fading channel employed and antenna design involved for achieving a reliable SIS approach. Below is the description of work with achievable EC of FD communications while considering passive and active SIS approaches.

\section{A. Passive Self-Interference Suppression Approach}

To mitigate SI through passive SIS approach, signals are treated with some antenna separation, shielding, and polarization before the signal actually enters a local transmitter. In [292], authors have analysed the arrival/source rate of FD two hop networks using the EC concept with certain delaybound. SI is minimized with the help of passive SIS approach while taking into consideration various buffer constraints (with respect to arrival rates) at relay, source, and destination node. 
TABLE V

ACHIEVABLE EFFECTIVE CAPACITY IN FD COMMUNICATIONS CAN BE STUDIED WHILE CONSIDERING DIFFERENT SIS APPROACHES, NETWORK TYPES, FADING CHANNELS, AND ANTENNA REQUIREMENTS

\begin{tabular}{|c|c|c|c|c|c|}
\hline \multicolumn{2}{|l|}{ SIS Approaches } & Study & Network Type & Fading Channel Used & Antenna Design \\
\hline \multirow[t]{7}{*}{$\begin{array}{l}\text { Active } \\
\text { Approaches }\end{array}$} & $\begin{array}{ll}\text { Digital } & \text { SIS } \\
\text { Approach } & \end{array}$ & [170] & FD-Relay Networks & Rayleigh Fading Channel & Not Defined \\
\hline & $\begin{array}{l}\text { Analog } \\
\text { Approach }\end{array}$ & [290] & FD-Relay Networks & Rayleigh Fading Channel & Two Antennas \\
\hline & \multirow[t]{5}{*}{$\begin{array}{l}\text { Analog and Digital } \\
\text { SIS Approach }\end{array}$} & [31] & FD-Relay Networks & Rayleigh Fading Channel & Single Antenna \\
\hline & & [62] & FD-Relay Networks & Nakagami- $m$ Fading Channel & Single Antenna \\
\hline & & [107] & $\begin{array}{l}\text { FD-Cognitive } \\
\text { Networks }\end{array}$ & Nakagami- $m$ Fading Channel & Not Defined \\
\hline & & [165] & FD-Relay Networks & Rayleigh Fading Channel & Not Defined \\
\hline & & [291] & FD-Relay Networks & Rayleigh Fading Channel & Not Defined \\
\hline \multirow{8}{*}{\multicolumn{2}{|c|}{ Passive SIS Approaches }} & [292] & FD-Relay Networks & Rayleigh Fading Channel & Directional Antenna \\
\hline & & [59] & FD-Relay Networks & Rayleigh Fading Channel & Single Antenna \\
\hline & & [61] & FD-Cellular Networks & Nakagami- $m$ Fading Channel & MIMO Antenna \\
\hline & & [113] & FD-Cellular Networks & Nakagami- $m$ Fading Channel & MIMO Antenna \\
\hline & & [33] & FD-Relay Networks & Nakagami- $m$ Fading Channel & $\begin{array}{l}\text { Bidirectional } \\
\text { Antenna }\end{array}$ \\
\hline & & [293] & FD-Relay Networks & Rayleigh Fading Channel & Not Defined \\
\hline & & [34] & FD-Relay Networks & Rayleigh Fading Channel & MIMO Antenna \\
\hline & & [294] & FD-Relay Networks & Rayleigh Fading Channel & MIMO Antenna \\
\hline \multirow{2}{*}{\multicolumn{2}{|c|}{ Hybrid SIS Approaches }} & [32] & FD-Relay Networks & Rayleigh Fading Channel & Two Antennas \\
\hline & & [295] & FD-Cellular Networks & Rayleigh Fading Channel & $\begin{array}{l}\text { Omni-directional } \\
\text { Antenna }\end{array}$ \\
\hline
\end{tabular}

Performance of the proposed scheme has been tested with achievable EC and shows improvement as compared to other state-of-the-art schemes. Time-critical and delay-sensitive applications such as video transmission with FD communications is investigated in [59], while considering the EC model. To predict the quality of the video streams, EC metric is used for detailed analysis with passive SIS approach. Passive SIS approach with delay constraints in this FD communications is compared with other two sub-optimal strategies.

Statistical QoS provisioning with the EC model has also been studied in emerging wireless networks such as FDenabled 5G networks [61]. In this study, passive SIS approach with MIMO antennas is used to suppress the SI by adjusting the transmission power. With passive SIS approach, Quadrature-OFDMA (Q-OFDMA) scheme with D2D communications has been analyzed with EC under stringent delay requirements. In this study, the maximization in EC has been studied under Nakagami- $m$ fading conditions. Authors in [113], have also used the concept of EC with FD-enabled 5G networks while using passive SIS approach. In this scheme, heterogeneous statistical QoS provisioning in 5G networks has been studied based on three network architectures namely, FD architecture, CRNs, and D2D network.

EC-based delay analysis with advance passive SIS approaches such as local transmit power unrelated selfinterference (LTPUS) and local transmit power related selfinterference (LTPRS) in FD communications have been discussed in [33]. In LTPUS, SIS approach does not directly depend on a power level, however in LTPRS the power of local transmitter is also considered to mitigate an excessive SI. Arrival rate in conjunction with SIS approach with required delay-outage probability has been analyzed using EC concept. Cross-layer resource allocation while using the SIS approach in FD communications with achievable EC has been discussed in [293]. In this study, SI has been overcome by optimally controlling power and by using the efficient relaying scheme. AF method of relay selection with EC concept has been analysed in detail. Proposed scheme has also been evaluated through extensive simulations and compared with a traditional FD scheme with direct transmission. EC-maximization shows that, proposed work shows two-fold improvement in throughput as compared to the other state-of-the-art work.

Statistical QoS provisioning with passive SIS approach based on buffer-aided relaying method in FD relay networks has been explored in [34]. SI has been controlled by optimally controlling transmit power of a local transmitter. EC framework in this scheme has been used to investigate the resource allocation scheme for optimally assigning the power. Relaymode selection criteria while utilizing the two-way MIMO systems with EC has been researched in [294]. Throughput under various QoS constraints and optimal selection of halfduplex or FD mode has been analysed with achievable EC. Proposed scheme shows better performance at low signal-tonoise ratio (SNR) with FD mode. However, at high SNR, halfduplex scheme outperforms the FD mode. These comparisons have been made while employing the EC model for FD mode.

\section{B. Active Self-Interference Suppression Approach}

Baseband canceller and RF canceller are used to actively mitigate SI at a local transmitter and receiver. Active SIS approaches in FD communications with EC has been discussed in literature. Through a proper implementation of active SIS approach, 40-50 dB of SI can be reduced. Achievable EC in FD communications while considering active SIS approach can be studied by classifying the active SIS approaches into digital, analog, and combination of these approaches.

1) Digital Self-Interference Suppression Approach: Nonlinearities in analog-to-digital converter (ADC) and irregularities in an oscillator can result into SI. SI resulting from such factors can be mitigated with the help of digital SIS approach. Dynamic range of a receiver ADC, can also be handled with the digital SIS approach. Authors in [170], 
have used the concept of EC to understand and model the digital SIS approach in two hop networks with FD relays. In this work, decode-and-forward (DF) relaying method has been used to support the cooperative communications. Both the source and relay queues, with certain delay-bound have been investigated with the EC model. A trade-off between source and relay queues regarding statistical QoS provisioning has been achieved while actively suppressing SI at relay node. Simulations have been performed to validate the ECbased mathematical framework for this SIS approach for FD networks. The EC analysis shows that the tradeoff between the two queues improves the sum of the effective capacity. This framwork also provides another insights regarding the importance of buffers at the relay. The relays with buffer shows better performance as compared to the relays without buffers.

2) Analog Self-Interference Suppression Approach: Complexities at ADC can also introduce SI. This SI can be effectively suppressed with the help of analog SIS approach. Various techniques such as time-domain algorithms, sequencebased methods or adaptive interference suppression have been introduced to actively suppress the SI. Analog SIS approach with optimal power allocation under channel uncertainties while using the concept of EC has been discussed in [290]. In this work, not only SI is suppressed but also the loopinterference has been minimized with stringent delay requirement. Problem of SI, loop interference, and optimal power allocation has been formulated by employing Taylor optimization and solved using Lagrange dual approach. Proposed scheme has been investigated with EC and shows improved performance as compared to the other state-of-the-art schemes while residing within statistical delay QoS constraints.

3) Analog and Digital Self-Interference Suppression Approach: Combination of analog and digital SIS approach is also used to mitigate SI that can result from the complexities, non-linearities in ADC, and irregularities in oscillator. This analog and digital SIS approach with achievable EC has also been investigated in the existing work. Analog and digital SIS approach in FD relay networks with buffer-aided relaying scheme with EC has been discussed in [31]. Infinite size queues at a source and a relay node has been taken into consideration with Rayleigh fading channel. In this work, EC is used to find the arrival/source rate, and depending upon the source rate, the relaying is performed, thats why this scheme has been named as "selection relaying".

Achievable EC with analog and digital SIS approach has also been studied with heterogeneous QoS requirements [62]. In this FD-relay networks, DF relaying scheme with Nakagami- $m$ fading channel has been investigated with EC. In this work, heterogeneous QoS-aware resource allocation scheme has been developed with the help of EC. Concept of EC with both analog and digital SIS approach has also been explored in FD-CRNs [107]. In this scheme, simultaneous spectrum sensing and transmission in CRNs in conjunction with statistical QoS provisioning has been modeled with EC. In this scheme, Nakagami- $m$ fading channel has been used and probabilities of false alarm and miss detection are derived. An in-depth EC-based QoS analysis with proposed FD-CRNs has been carried out to study the required delay-violation probability for real-time applications.

Another buffer-aided relay selection scheme in FD-relay networks with analog and digital SIS approach has been explored in detail using EC concept in [165]. A trade-off between statistical delay constraints on two concatenated queues has been derived. Then a maximum constant arrival rate is derived using the EC model. SI at the FD-relay has been minimized while considering analog and digital SIS approach with Rayleigh fading channel. EC-based performance analysis shows that proposed scheme shows an improved throughput as compared to other state-of-the-art schemes. Achievable EC with analog and digital SIS approach has also been studied in FD-relay networks with AF relaying protocol [291]. This work provides the idea of better link quality selection between a source and relay node to support the desired QoS requirements for FD-relay networks. Link quality between a source and relay node has been investigated while considering the EC model. This analysis shows that the SIS approaches also affect the link quality in FD relay networks.

\section{Hybrid Self-Interference Suppression Approach}

In some cases, both passive and active SIS approaches are utilized to completely suppress SI. With hybrid SIS approach, concept of EC has also been employed to investigate the proposed SI approach. Authors in [32], have used the concept of EC for FD-relay networks with hybrid SIS approach. In this study, both AF and DF relaying schemes with their FD and half-duplex support have been discussed in detail. Further, a new control factor named as the cancellation coefficient has been proposed and both the half-duplex and FD mode has been analyzed with achievable EC. Extensive analysis based on EC concept reveals that, hybrid mode comprising of halfduplex and FD operation shows better performance. Hybrid SIS approach with the EC model has also been studied in FD-cellular networks [295]. SI resulting from omni-directional antenna has been mitigated with the help of passive and analog SIS approach. Whole network with hybrid SIS approach has been modeled as a Matern point process and then is analysed with the EC concept.

\section{Summary and Insights}

Achievable EC in FD-communications with SIS approaches has been reviewed in this section. Simultaneous transmission and reception (FD communications) with stringent QoS provisioning is a challenging task. The EC based analsyis of FDrelays provides important insight regarding the provision of buffer at the relay. The FD relays with buffers shows improved performance as compared to the relays without buffers. Investigating the achievable EC of FD communications with other networks such as CRNs and cellular networks further adds the complexity. Taking the closed-form expression of EC with FD communications is more complex than ergodic and Shannon capacity. Designing of resource allocation schemes with proper SIS approaches for FD communications invites the future researchers to explore this dimension with achievable EC. 

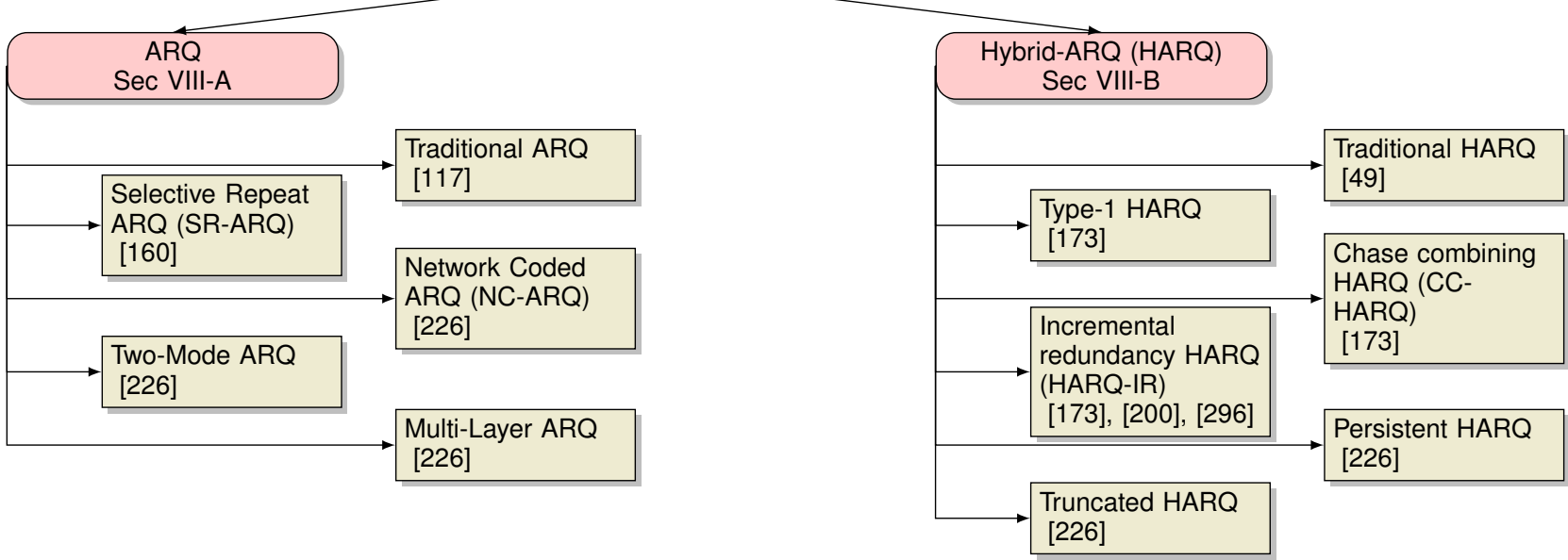

Fig. 5. Existing work on effective capacity while employing retransmission schemes are based on different flavors of ARQ and Hybrid-ARQ (HARQ)

\section{EFFECTIVE CAPACITY AND Re-TRANSMISSION SCHEMES}

Packet switched networks are often prone to packet loss. Therefore, packet retransmission schemes are employed to achieve reliable communications in these networks. Two versions of packet retransmission schemes, namely, automatic repeat request (ARQ) and hybrid automatic repeat request (HARQ), are extensively used to achieve a better reliability in the networks. These retransmission schemes use different forms of acknowledgement mechanisms to recover the loss packets in a network. Achievable EC for the various retransmission schemes has been discussed in the existing literature. In this paper, current work on retransmission schemes with achievable EC has been classified into ARQ and HARQ. Figure 5, shows a classification of existing work on retransmission schemes that use EC concept. Different versions of ARQ and HARQ with EC have also been surveyed in this Section. Below is the description of retransmission schemes that considers the EC model.

\section{A. Automatic Repeat Request (ARQ)}

ARQ is extensively used to achieve the required reliability in networks. Concept of EC in conjunction with ARQ has been used to anaylse the statistical delay provisioning while residing within certain QoS constraints. The achievable EC of various versions of ARQ ranging from traditional to two-mode ARQ have also been explored. Details of some of these versions are provided below:

1) Traditional ARQ: Authors in [117], have discussed the EC maximization with adaptive modulation and coding (AMC) and ARQ schemes in underlay CRNs. In this study, an optimal power allocation scheme has been discussed while meeting the stringent requirements of packet-error rate (PER) and delay constraints. Power requirements and throughput were also analysed by using the EC model.
2) Selective Repeat $A R Q$ (SR-ARQ): ARQ with finite retranmission persistence scheme such as truncated ARQ are utilized to recover the lost packets in lossy links. However, as compared to finite retranmission persistence, ARQ with infinite persistence can also be used to achieve the required reliability in network. The achievable EC of ARQ methods with infinite persistent named as selective repeat ARQ (SRARQ) has been analysed in [160]. In this work, performance of SR-ARQ has been analyzed in two approaches. First, a discrete-time Markov chain (DTMC) model is used to analyze the queuing behavior and arrival process. In the second approach, concept of effective bandwidth and EC are used to find the maximum arrival/source rate with desired delay-bound with infinite persistent SR-ARQ approach.

3) Network Coded $A R Q$ (NC-ARQ): Concept of network coding (NC) has been extensively used to optimize the flow of digital data in a network. To communicate multiple packets over the same time slot, concept of network-coded ARQ (NCARQ) has been proposed. Authors in [226], have provided a comparative view of different retransmissions schemes while considering the concept of EC model. In this study, NC-ARQ has been proposed and compared with other versions of ARQ and HARQ. To achieve the reliability in transmission, multiple packets are transmitted in a single stream. Performance analysis of NC-ARQ based on achievable EC, shows that NC-ARQ outperforms the other state-of-the-art retransmission schemes.

4) Two-Mode ARQ: In [226], the maximization of $\mathrm{EC}$ with two-mode ARQ in comparison to traditional ARQ with Gilbert-Elliot block fading channel has been investigated in detail. In this two-states or two-mode ARQ, a channel always assumes either of the two states and these two states at any time can be, bad-to-good, bad-to-bad, good-to-bad, and goodto-good. During, each mode or state, achievable EC has been investigated for the proposed ARQ with various retransmission parameters.

5) Multi-Layer ARQ: Similar to the idea of NC-ARQ, multi-layer ARQ can also transmit multiple packets in a sin- 
gle transmission [226]. By adjusting the transmission power, various codewords are communicated in a single stream. Receivers are intelligently designed to decode these codewords by considering the channel fading conditions. Performance of multi-layer ARQ has been anaylsed with EC to find the maximum arrival/source rate with given delay requirement.

\section{B. Hybrid Automatic Repeat Request (HARQ)}

In wireless communications, reliability has been further strengthen with the introduction of HARQ. In HARQ, traditional ARQ error-control mechanism, has been further enhanced with the inclusion of high rate forward error correction (FEC) code. With the help of link-layer capacity model, i.e., EC, new versions of HARQ has also been analyzed while residing within certain delay constraints. Below is the description of various versions of HARQ in conjunctions with EC model.

1) Traditional HARQ: To deal with challenging conditions of dynamic wireless medium, different transmission schemes including the adaptive modulation coding (AMC) and HARQ have been introduced and then have been investigated with their achievable EC. Authors in [49], have provided a comparative view of AMC and HARQ while considering the EC model. Delay caused by AMC and HARQ has been taken into account to analyse the performance of the above both. Performance analysis based on EC maximization for HARQ and AMC shows that their performance decreases at high SNR.

2) Type-I HARQ: Type-I HARQ, is the simplest version of HARQ [173]. In type-I HARQ, during transmission of packets, error-detection (ED) and FEC mechanisms are added to each message to make transmissions of packets more reliable. In [173], type-I HARQ has been analysed with EC, while considering different transmission rates and delayconstraints. In this retransmission scheme, data packets are encoded into one codeword by using one of the channel code from a codebook. A codebook, carrying all the available channels is maintained and one codeword is transmitted in one time/frequency slot. This scheme, is then assessed using EC model. Extensive mathematical and simulation evaluation of the proposed scheme shows that, receiver can only decode codeword when transmission rate is low.

3) Chase Combining HARQ (CC-HARQ): EC of chase combining HARQ (CC-HARQ) scheme has been discussed in [173]. In this CC-HARQ scheme, power gain is achieved before decoding a packet. Repeated transmissions are achieved in each packet transmission. Performance of CC-HARQ has been compared with incremental redundancy HARQ (HARQIR) based on the concept of achievable EC. This analysis shows that, Type-I HARQ and CC-HARQ are the only two retransmission schemes that can perform repeated transmission in a single stream.

4) Incremental Redundancy HARQ (HARQ-IR): Type-II HARQ is also termed as an incremental redundancy HARQ (HARQ-IR). In this scheme, sender can select either FEC or error detecting parity bits. Work in [173], considers HARQIR with the concept of EC. A comparative view of HARQIR has also been provided with other versions of HARQ.
In HARQ-IR, a complex codeword is first abstracted into simple sub-codewords, and then is transmitted in consecutive time slots, untill the transmission deadline expires. In this study, the maximization of EC with HARQ-IR has been investigated with the required delay-outage probability. Authors in [200], [296], have provided an-depth analysis of HARQ-IR with delay-sensitive traffic while considering EC concept. Impact of retransmission and other advance schemes of physical layer on a data link layer especially the queuing performance has also been studied with the EC model. In this work, physical layer parameters (codewords of HARQ-IR) has been adjusted according to queuing requirements of delaysensitive traffic. The maximum arrival/source rate has been found with achievable EC to analyze the physical channel with required delay requirements. Statistical QoS provisioning with HARQ-IR in buffer-aided diamond relay systems has been discussed in [297]. Concept of outage effective capacity has been introduced to remove the packets from buffer that are not successfully received after a certain number of transmission attempts. This EC analysis shows that, the performance of HARQ-IR is same either using buffer-aided diamond relaying or decode-and forward relaying.

5) Persistent HARQ: Performance of persistent HARQ has also been tested while using EC concept [226]. In this technique, a transmitter persistently resends packets for a given duration of a time (frame). In this scheme, each retransmission scheme is accompanied by a reward and is analysed with achievable EC with delay requirements. It is the only study, that takes into consideration a detailed analysis of various versions of HARQ with EC concept.

6) Truncated HARQ: Authors in [226], have considered the mathematical framework based on EC for truncated HARQ. In truncated HARQ, an upper limit is imposed on the number of retransmission schemes. This upper limit is often termed as "transmission limit". With an upper limit on retransmission, excessive energy for excessive retransmission has been conserved. Performance of a truncated HARQ while considering "transmission limit" with maximum achievable EC has been investigated in detail with different delay-bounds.

\section{Summary and Insights}

In this section, an in-depth analysis of various retransmission schemes has been discussed while considering the EC model. However, to achieve the utlra-reliability and lowlatency, retransmission schemes have not been taken into account in some of the mission-critical applications of 5G, such as, URLLC [287]. To support these applications, finite code-length has been used instead of retransmission schemes. This can result into achieving the ultra-reliability and very low latency. Therefore, under the stringent delay requirements, in addition to the retransmission schemes, other vistas such as diversity [287] should be explored to achieve the given reliability.

\section{Open Issues, Challenges, and Future Research DIRECTIONS}

As the complexity or size of the networks increases, the mathematical modelling for the EC maximization also tends 
to become more difficult and complex. For example, the monotonicity and concavity for the achievable EC in multicarrier system and single-carrier system does not necessarily hold. This could pose a serious challenge for employing the EC to understand the performance of the different networks while providing the statistical QoS requirements.

Extensive work has been done on EC while considering various wireless networks. With the emergence of $5 \mathrm{G}$ and beyond, the provision of stringent delay requirements needs to be addressed very carefully. Different applications have different delay requirement (some require very low-delay bound). Using the EC to model the delay for various applications and wireless networks with various delay-bounds is a challenging task. Wireless networks have also their own limitations and constraints such as energy limitation in WSNs. Maximizing EC while residing within these limitations is even further a challenging task. After a careful overview of existing work on EC, we have realized that there are also good potential for the future researchers to use the concept of EC with other state-of-the-art technologies which have also been highlighted below:

\section{A. Effective Capacity and Ultra-reliable Low Latency Com- munications (URLLC)}

International Mobile Telecommunications (IMT)-advance usually concerned about the data rate. However, to accommodate the escalating demand of emerging future wireless networks, IMT-2020 provides the guidelines for the enhanced mobile broadband (eMBB), URLLC, and massive machine type communications (massive MTC). With latency requirement of $1 \mathrm{~ms}$, and $99.999 \%$ reliability, URLLC has gained much attention for the mission-critical applications such as Industry 4.0. To meet the stringent delay requirements of URLLC, transmission-delay and queueing-delay should be very small [298].

Authors in [16] have discussed the various tools/metrics such as effective bandwidth, extreme value theorem, and stochastic network calculus to investigate the techniques in URLLC. In URLLC, the main concern is all about the modelling of the factors with much accuracy that occur very rarely. Main benefit of using EC to model the delay in URLLC is its simple modelling of the link performance [6]. Ultra latency as well as the reliability have been modeled using the EC model and the short-length codes (short packet communications) in [299]. Two cases in which the instantaneous and statistical CSI that are available at the transmitter are considered to find the bounds on reliability and latency. Using the EC tool to investigation URLLC with short packet communications still needs further insights from researchers in academia and industry. Topics such as optimal resource allocation with short packet communications open the door for future research.

\section{B. Energy Harvesting Based EC Maximization}

For wireless networks, energy is a scarce commodity. Conserving energy in wireless networks, has been a prime goal for researchers [300]-[302]. With introduction of energyharvesting batteries or modules, performance of wireless networks can be improved [303]. To the best of authors's knowledge, very limited work has been done on investigating the effect of energy-harvesting on the achievable EC of wireless networks. Depending upon the energy-harvesting source (such as sun in case of solar energy) the arrival of energy-packets is uncertain or not fixed. Therefore, with the introduction of delay requirements this uncertain behavior of arrived energypackets can pose a serious challenge to guarantee the low delay communications. To address this challenge, EC can be used to model the stringent delay requirements and to investigate the different limitations of energy-harvesting system. This analysis can also help in changing and improving the design of energyharvesting modules and transmitters.

\section{Challenges Related to Effective Capacity Simulation}

As compared to the Shannon and ergodic capacity, simulation of the EC is tricky one especially in multi-carrier and multi-user case. As compared to the single user and singlecarrier, concavity as well as the monotonicity for EC does not hold in multiuser and multi-carrier systems [132]. Therefore, as the complexity of the network increases, the simulations of the EC becomes more and more challenging. Simulations, as well as the mathematical modelling considering the EC, become more complex while considering the complex network scenarios such as the case in heterogeneous networks.

Authors in [304] have proposed an EC estimation tool named as "CrEST". It is the theoretical EC estimation tool for single-hop and single-carrier systems. Different parameters for EC such as delay-bound, queue-length, and others are predefined and then the maximization in EC is estimated to assess the performance of the system. However, this tool do not take into consideration the EC maximization in multi-carrier and multi-user systems.

\section{Security and Privacy Issues in Low Latency Communica- tions}

Security is a strong pillar in wireless communication for transmitting common and confidential information. Any breach in the communication security can result into the damage to user data or user privacy. A well-known technique to provide security is to use cryptography, however because of security code bits, providing delay-limited communications is even more challenging. EC-based delay analysis in various wireless networks with different security threats has also been studied in [163], [290]. In normal scenarios, EC estimates the delay of information bits in the transmission-buffer. However, in cryptography, in addition to the information bits, there are also the coded bits in the information buffer. In this case, EC takes into consideration the delay of coded bits only, so the delay analysis of the information bits remains unattended. This opens the door for the future research to investigate delayanalysis while considering the cryptography techniques. 


\section{E. Effective Capacity of Cognitive Radio Networks (CRNs)}

In normal operation of CRNs, SUs have to wait for the PUs to vacate the channel. This can result into some uncertain or undefined delay depending on the PUs activity patterns. In scenarios where the SUs have also to support the delaysensitive applications, then the combination of this delay requirement and the undefined delay for accessing the channel can complicate the situation. This challenge needs to be addressed by carefully analysing the delay and performance of the system. For this purpose, EC has been extensively used to understand the delay analysis of the CRNs.

1) Spectrum Sensing in CRNs and Effective Capacity: Among EC-based wireless networks, CRNs have been studied and analyzed extensively with EC metric. Efficient spectrum sensing in CRNs can enhance the performance of a system. Transmission of QoS-aware applications in CRNs requires efficient sensing and transmission on the part of SUs [198]. Depending on the delay requirements and the PUs activity patterns, in-depth analysis can be performed with the help of EC. This delay-analysis can further be used to design the QoSaware spectrum sensing approaches to facilitate the delaysensitive applications.

2) White Space Utilization in CRNs and Effective Capacity: Depending on white space utilization, CRNs can further be classified into underlay, overlay, interweave, and hybrid CRNs. Most of the existing work on performance evaluations based on EC metric in CRNs only considers underlay and overlay CRNs. Depending on the delay requirements, which approach best fits for different delay-sensitive applications should be explored further and this challenge invites the future researchers to investigate further into this domain.

3) Spectrum Mobility and TV white space in CRNs and Effective Capacity: In CRNs, simplification of quantification between spectrum mobility, TV white space, spectrum sensing, delay, and PUs activity patterns is a challenging task. Authors in [305], discuss that, the above mentioned challenge of quantification can be addressed with the help of EC. EC helps in simplifying the performance of the system, which can be used to understand the trade-off between different techniques in CRNs.

\section{F. Challenges Related to Lincensed-Assisted Access in Unli- censed Spectrum}

Licensed-assisted access (LAA) in unlicensed band has been witnessed as a promising solution to mitigate the current spectrum crunch for escalating demand of cellular traffic. Provision of QoS in LAA is a challenging task. This challenge is due to the dynamic and heterogeneous nature of LAA and co-existence of the wifi networks. QoS provisioning in LAA can be simplified with the help of EC as has been discussed in [306]. This EC-based performance analysis invites the future researchers to explore the new vistas related to the unlicensed spectrum sensing, spectrum-mobility, and unlicensed users activity to avoid the interference.

\section{G. Optimal Power and Resource Allocation with Effective Capacity model}

Resource allocation for multi-user and multi-carrier systems while considering the EC model can be challenging. For single-carrier and multi-carrier, monotonicity and concavity of EC does not necessarily hold. Therefore, with the EC model, resource allocation strategies for single-carrier systems cannot be simply used for multi-carrier systems [132]. Resource allocation with stringent-delay requirements is also a challenging task for the emerging wireless networks. QoS provisioning with optimal resource allocation for the cell-edge users have not been anaylsed in detail [307]. Work in [307], is the first attempt to understand the delay requirements for the cell edge users in multi-cell heterogeneous networks.

\section{H. Effective Capacity of Heterogeneous Networks}

Heterogeneous networks consist of very diverse network components/architecture. These networks have also had to support the diverse delay-sensitive applications with different delay requirements. Therefore, provisioning and analysing the delay for the heterogeneous networks is a challenging task. EC is a useful tool that can simplify the performance analysis of the complex and diverse networks such as heterogeneous networks. EC is a flexible tool that can also provide the clean slate to understand the different delay requirements (with different delay-bound) for the diverse range of networks [6].

\section{Conclusion}

Quality of service (QoS)-aware and delay-sensitive realtime applications require wireless channel models that can incorporate QoS-aware evaluation metrics such as delay, data rate, and delay-violation probability. Existing physical-layer channel models do not consider QoS metrics. To address this issue, QoS-aware link-layer wireless channel model named as "effective capacity (EC)" has been proposed. In this paper, we have provided a comprehensive survey of the EC model with its state-of-the-art work in different wireless networks. How EC metric can be used for testing the performance of various wireless networks, has been surveyed in detail in this paper. Five different case studies involving EC concept in cellular networks, device-to-device (D2D) communications, full-duplex (FD) communications, peer-to-peer streaming, and visible light communications have been presented. Various QoS-aware and delay-sensitive applications such as voice, video, and medical applications analysed by the EC model have also been surveyed. EC-based delay analysis in wireless networks under different fading models such as stochastic, generalized, dimension-based, supplementary, and futuristic fading models have been provided. Among these fading channels Rayleigh fading model has been extensively evaluated with EC metric. Concept of EC in different networks such as CRNs, relay networks, cellular networks, mesh networks, and adhoc networks have been explored in detail. This paper also covers the achievable EC in FD communications and various retransmission schemes. In the last, we have concluded this paper by outlining some open issues and future research directions related to the EC model in various existing and advance future wireless networks. 


\section{REFERENCES}

[1] C. Zhong, T. Ratnarajah, K.-K. Wong, and M.-S. Alouini, "Effective capacity of multiple antenna channels: Correlation and keyhole," IET communications, vol. 6, no. 12, pp. 1757-1768, 2012.

[2] Q. Du and X. Zhang, "Base-station selections for QoS provisioning over distributed multi-user MIMO links in wireless networks," in IEEE INFOCOM, 2011, pp. 3038-3046.

[3] — , "QoS-aware base-station selections for distributed MIMO links in broadband wireless networks," IEEE Journal on Selected Areas in Communications, vol. 29, no. 6, pp. 1123-1138, 2011.

[4] M. Amjad, M. H. Rehmani, and S. Mao, "Wireless multimedia cognitive radio networks: A comprehensive survey," IEEE Communications Surveys Tutorials, 2018.

[5] M. G. Khoshkholgh, K. Navaie, K. G. Shin, and V. CM Leung, "Provisioning statistical QoS for coordinated communications with limited feedback," in IEEE Global Communications Conference (GLOBECOM), 2016

[6] D. Wu and R. Negi, "Effective capacity: a wireless link model for support of quality of service," IEEE Transactions on wireless communications, vol. 2, no. 4, pp. 630-643, 2003.

[7] X. Guo, L. Dong, Y. Li, and L. Wang, "Effective capacity of mimo mrc system with constant and variable power loading," in 13th Canadian Workshop on Information Theory (CWIT), 2013, pp. 117-121.

[8] K. Angrishi and U. Killat, "Analysis of a real-time network using statistical network calculus with effective bandwidth and effective capacity," in 14th GI/ITG Conference Measuring, Modelling and Evaluation of Computer and Communication Systems (MMB), 2008, pp. 1-15.

[9] K. Angrishi, "An end-to-end stochastic network calculus with effective bandwidth and effective capacity," Computer Networks, vol. 57, no. 1, pp. 78-84, 2013.

[10] M. Hammouda, S. Akin, and J. Peissig, "Effective capacity in multiple access channels with arbitrary inputs," in IEEE 11th International Conference on Wireless and Mobile Computing, Networking and Communications (WiMob), 2015, pp. 406-413.

[11] C. Wang, B. Urgaonkar, A. Gupta, L. Y. Chen, R. Birke, and G. Kesidis, "Effective capacity modulation as an explicit control knob for public cloud profitability," in IEEE International Conference on Autonomic Computing (ICAC), 2016, pp. 95-104.

[12] Z. Feng, G. Wen, and C. W. Chen, "Multiuser effective capacity analysis for queue length based rate maximum wireless scheduling," in 1st IEEE International Conference on Communications in China (ICCC), 2012, pp. 438-442.

[13] D. Qiao and M. C. Gursoy, "Buffer-aided relay systems under delay constraints: Potentials and challenges," IEEE Communications Magazine, 2017.

[14] C. Shen, Z. Yu, C. W. Chen, and F. Wu, "On the effective capacities of distributed and co-located large-scale antenna systems," in IEEE International Conference onCommunications (ICC). IEEE, 2017, pp. $1-6$.

[15] Y. Gao, N. Li, J. Zhang, and K. Xu, "Effective capacity of cognitive radio systems," in IEEE 13th International Conference on Signal Processing (ICSP), 2016, pp. 1757-1761.

[16] M. Bennis, M. Debbah, and H. V. Poor, "Ultra-reliable and lowlatency wireless communication: Tail, risk and scale," arXiv preprint arXiv:1801.01270, 2018.

[17] C. She, C. Yang, and T. Q. Quek, "Cross-layer optimization for ultrareliable and low-latency radio access networks," IEEE Transactions on Wireless Communications, vol. 17, no. 1, pp. 127-141, 2018.

[18] C.-S. Chang and T. Zajic, "Effective bandwidths of departure processes from queues with time varying capacities," in IEEE Fourteenth Annual Joint Conference of the IEEE Computer and Communications Societies. Bringing Information to People NFOCOM'95, vol. 3, 1995, pp. 10011009.

[19] C.-S. Chang, Performance guarantees in communication networks. Springer Science \& Business Media, 2012.

[20] _ Performance guarantees in communication networks. Springer Science \& Business Media, 2012.

[21] Q. Du and X. Zhang, "Resource allocation for downlink statistical multiuser QoS provisionings in cellular wireless networks," in IEEE INFOCOM, 2008, pp. 2405-2413.

[22] S. Glisic, Z. Nikolic, N. Milosevic, and P. Pirinnen, "Effective capacity of advanced wireless cellular networks," in IEEE 16th International Symposium on Personal, Indoor and Mobile Radio Communications (PIMRC), vol. 4, 2005, pp. 2771-2780.
[23] D. Wu and R. Negi, "Downlink scheduling in a cellular network for quality-of-service assurance," IEEE Transactions on Vehicular Technology, vol. 53, no. 5, pp. 1547-1557, 2004.

[24] S. W. H. Shah, M. M. U. Rahman, A. N. Mian, A. Imran, S. Mumtaz, and O. A. Dobre, "On the impact of mode selection on effective capacity of device-to-device communication," IEEE Wireless Communications Letters, pp. 1-1, 2019.

[25] C. Xiao, J. Zeng, W. Ni, R. P. Liu, X. Su, and J. Wang, "Delay guarantee and effective capacity of downlink noma fading channels," IEEE Journal of Selected Topics in Signal Processing, 2019.

[26] X. Han, H. Chen, L. Xie, and K. Wang, "Effective capacity region in a wireless multiuser OFDMA network," in IEEE Global Communications Conference (GLOBECOM), 2012, pp. 1794-1799.

[27] X. Hailun, W. Ning, and Z. Zhimin, "Neighbour peer selection scheme based on effective capacity for mobile peer-to-peer streaming," China Communications, vol. 10, no. 5, pp. 89-98, 2013.

[28] F. Jin, X. Li, R. Zhang, C. Dong, and L. Hanzo, "Resource allocation under delay-guarantee constraints for visible-light communication," IEEE Access, vol. 4, pp. 7301-7312, 2016.

[29] F. Jin, R. Zhang, and L. Hanzo, "Resource allocation under delayguarantee constraints for heterogeneous visible-light and RF femtocell," IEEE Transactions on Wireless Communications, vol. 14, no. 2, pp. 1020-1034, 2015.

[30] X. Li, F. Jin, R. Zhang, and L. Hanzo, "Joint cluster formation and user association under delay guarantees in visible-light networks," in IEEE Global Communications Conference (GLOBECOM), 2016, pp. $1-6$.

[31] D. Qiao, "Effective capacity of buffer-aided full-duplex relay systems with selection relaying," IEEE Transactions on Communications, vol. 64 , no. 1 , pp. 117-129, 2016.

[32] W. Cheng, X. Zhang, and H. Zhang, "Full/half duplex based resource allocations for statistical quality of service provisioning in wireless relay networks," in IEEE INFOCOM, 2012, pp. 864-872.

[33] _ , "QoS driven power allocation over full-duplex wireless links," in IEEE International Conference on Communications (ICC), 2012, pp. $5286-5290$.

[34] K. T. Phan and T. Le-Ngoc, "Buffer-aided full-duplex relaying with residual self-interference and statistical delay provisioning," in IEEE 26th Annual International Symposium on Personal, Indoor, and Mobile Radio Communications (PIMRC), 2015, pp. 250-255.

[35] S. w. Ahn, H. Wang, and D. Hong, "Throughput-delay tradeoff of proportional fair scheduling in OFDMA systems," IEEE Transactions on Vehicular Technology, no. 9, pp. 4620-4626.

[36] P. Parag, S. Sah, S. Shakkottai, and J. F. Chamberland, "Value-aware resource allocation for service guarantees in networks," IEEE Journa on Selected Areas in Communications, vol. 29, no. 5, pp. 960-968, 2011.

[37] S. Y. Lien, C. C. Tseng, K. C. Chen, and C. W. Su, "Cognitive radio resource management for QoS guarantees in autonomous femtocell networks," in IEEE International Conference on Communications (ICC), 2010, pp. 1-6.

[38] P. Parag, S. Shakkottai, and J. F. Chamberland, "Value-aware resource allocation for service guarantees in networks," in IEEE INFOCOM, 2010, pp. 1-9.

[39] X. Zhang and Q. Du, "Cross-layer modeling for QoS-driven multimedia multicast/broadcast over fading channels in [advances in mobile multimedia]," IEEE Communications Magazine, vol. 45, no. 8, pp. 62-70, 2007.

[40] S. Khurana, G. T. Kim, M. Elaoud, S. Samtani, and A. Staikos, "Performance evaluation of a unicast routing control agent for proactive diverse link selection," in IEEE Military Communications conference (MILCOM), 2006, pp. 1-7.

[41] A. D. Maleki and B. Abolhassani, "New scheduling scheme for green communications in long term evolution networks," IET Communications, vol. 8, no. 14, pp. 2438-2444, 2014.

[42] M. Matthaiou, G. C. Alexandropoulos, H. Q. Ngo, and E. G. Larsson, "Effective rate analysis of MISO rician fading channels," in IEEE 7th Sensor Array and Multichannel Signal Processing Workshop (SAM), 2012, pp. 53-56.

[43] Q. Wang, P. Fan, D. O. Wu, and K. B. Letaief, "End-to-end delay constrained routing and scheduling for wireless sensor networks," in IEEE International Conference on Communications (ICC), 2011, pp. $1-5$.

[44] S. Y. Lien, Y. Y. Lin, and K. C. Chen, "Cognitive and game-theoretical radio resource management for autonomous femtocells with QoS guarantees," IEEE Transactions on Wireless Communications, vol. 10, no. 7 , pp. 2196-2206, 2011. 
[45] Y. Chen and I. Darwazeh, "End-to-end delay performance analysis in IEEE 802.16j mobile multi-hop relay (mmr) networks," in 18th International Conference on Telecommunications, 2011, pp. 488-492.

[46] Y. Chen, I. Darwazeh, N. Philip, and R. Istepanian, "End-to-end delay distributions in wireless tele-ultrasonography medical systems," in IEEE Global Communications Conference (GLOBECOM), 2013, pp. 2592-2597.

[47] M. You, X. Mou, and H. Sun, "Effective capacity analysis of smart grid communication networks," in IEEE 20th International Workshop on Computer Aided Modelling and Design of Communication Links and Networks (CAMAD), 2015, pp. 196-200.

[48] W. Wang, S. Ci, H. Wang, D. Peng, and H. Sharif, "Quality-based adaptive image transmission in multihop wireless mesh networks," in Second International Conference on Communications and Networking in China, 2007, pp. 942-946.

[49] R. Sassioui, L. Szczecinski, L. Le, and M. Benjillali, "AMC and HARQ: effective capacity analysis," in IEEE Wireless Communications and Networking Conference (WCNC), 2016, pp. 1-7.

[50] Y. Chen, L. Dong, and I. Darwazeh, "Effective capacity-based delay performance estimators for lte radio bearer QoS provision," in 9th International Symposium on Communication Systems, Networks \& Digital Signal Processing (CSNDSP), 2014, pp. 105-110.

[51] M. Lari, A. Mohammadi, A. Abdipour, and I. Lee, "Characterization of effective capacity in antenna selection MIMO systems," journal of communications and networks, vol. 15, no. 5, pp. 476-485, 2013.

[52] S. Aksu and G. K. Kurt, "Analysis of effective capacity under approximate composite fading," in 21st Signal Processing and Communications Applications Conference (SIU), 2013, pp. 1-4.

[53] E. A. Jorswieck, R. Mochaourab, and M. Mittelbach, "Effective capacity maximization in multi-antenna channels with covariance feedback," IEEE Transactions on Wireless Communications, vol. 9, no. 10, pp. 2988-2993, 2010.

[54] B. Soret, M. C. Aguayo-Torres, and J. T. Entrambasaguas, "Capacity with explicit delay guarantees for generic sources over correlated rayleigh channel," IEEE Transactions on Wireless Communications, vol. 9 , no. 6, 2010.

[55] C. Amo-Quarm and K. Sohraby, "The effect of initial on-state probability on effective capacity," in International Zurich Seminar on Broadband Communications, Access, Transmission, and Networking, 2002, pp. 17-17.

[56] A. A. Khalek, C. Caramanis, and R. W. Heath, "Delay-constrained video transmission: Quality-driven resource allocation and scheduling," IEEE Journal of Selected Topics in Signal Processing, vol. 9, no. 1, pp. 60-75, 2015

[57] X. Zhang and J. Wang, "Statistical QoS-driven power allocation for wifi offloading over heterogeneous wireless networks," in Information Sciences and Systems (CISS), 2017 51st Annual Conference on, 2017, pp. 1-6.

[58] - "Heterogeneous QoS-driven resource allocation over MIMOOFDMA based 5G cognitive radio networks," in IEEE Wireless Communications and Networking Conference (WCNC), 2017, pp. 1-6.

[59] C. Ye, M. C. Gursoy, and S. Velipasalar, "Optimal resource allocation for full-duplex wireless video transmissions under delay constraints," in IEEE Wireless Communications and Networking Conference (WCNC), 2017, pp. 1-6.

[60] J. Wang and X. Zhang, "Statistical QoS-driven cooperative power allocation game over wireless cognitive radio networks," in IEEE Wireless Communications and Networking Conference (WCNC), 2017, pp. 1-6.

[61] X. Zhang and J. Wang, "Statistical QoS-driven power adaptation over Q-OFDMA-based full-duplex D2D 5G mobile wireless networks," in IEEE Wireless Communications and Networking Conference (WCNC), 2017, pp. 1-6.

[62] J. Wang and X. Zhang, "Heterogeneous QoS-driven resource adaptation over full-duplex relay networks," in IEEE Global Communications Conference (GLOBECOM), 2016, pp. 1-6.

[63] H. Hosseiny, M. Baniasadi, V. Shah-Mansouri, and M. Ghanbari, "Power allocation for statistically delay constrained video streaming in femtocell networks based on Nash bargaining game," in IEEE 27th Annual International Symposium on Personal, Indoor, and Mobile Radio Communications (PIMRC), 2016, pp. 1-6.

[64] S. V. Azhari, O. Gurbuz, and O. Ercetin, "QoS based aggregation in high speed IEEE802. 11 wireless networks," in Mediterranean Ad Hoc Networking Workshop (Med-Hoc-Net), 2016, pp. 1-7.

[65] C. She, C. Yang, and L. Liu, "Energy-efficient resource allocation for MIMO-OFDM systems serving random sources with statistical QoS requirement," IEEE Transactions on Communications, vol. 63, no. 11, pp. 4125-4141, 2015.

[66] M. Fidler, R. Lübben, and N. Becker, "Capacity-delay-error boundaries: A composable model of sources and systems," IEEE Transactions on Wireless Communications, vol. 14, no. 3, pp. 1280-1294, 2015.

[67] J. Li, W. Xia, P. Martins, and L. Shen, "Low complexity user scheduling for multi-antenna gaussian broadcast systems with quality of service requirements," IET Communications, vol. 8, no. 10, pp. 1820-1830, 2014.

[68] A. A. Khalek, C. Caramanis, and R. W. Heath, "Video qualitymaximizing resource allocation and scheduling with statistical delay guarantees," in IEEE Global Communications Conference (GLOBECOM), 2013, pp. 1736-1740.

[69] R. Chen, R. Q. Hu, Q. Li, and G. Wu, "Energy efficiency in a delay constrained wireless network," in IEEE Wireless Communications and Networking Conference (WCNC), 2013, pp. 836-841.

[70] Y. Chen and I. Darwazeh, "An estimator for delay distributions in packet-based wireless digital communication systems," in IEEE Wireless Communications and Networking Conference (WCNC), 2013, pp. 825-829.

[71] Z. Feng and G. Wen, "QoS guarantees of multiuser video streaming over wireless links: Delay constraint and packet priority drop," China Communications, vol. 10, no. 3, pp. 133-144, 2013.

[72] Z. Feng, G. Wen, Z. Zou, and C. W. Chen, "Wireless video streaming QoS guarantees based on virtual leaky bucket," in IEEE Global Telecommunications Conference (GLOBECOM), 2011, pp. 1-6.

[73] S. Ahn, S. Lee, K. Kim, and D. Hong, "Analysis of proportional fair scheduling in OFDMA systems with delay QoS constraints," in 16th Asia-Pacific Conference on Communications (APCC), 2010, pp. 255260.

[74] Q. Wang, D. O. Wu, and P. Fan, "Delay-constrained optimal link scheduling in wireless sensor networks," IEEE Transactions on Vehicular Technology, vol. 59, no. 9, pp. 4564-4577, 2010.

[75] S. Ahn, H. Wang, K. Kim, and D. Hong, "The effect of multiplexing users in QoS provisioning scheduling," in Sixth Advanced International Conference on Telecommunications, 2010, pp. 491-496.

[76] M. Li, M. Claypool, and R. Kinicki, "WBest: a bandwidth estimation tool for IEEE 802.11 wireless networks," in IEEE Conference on Local Computer Networks (LCN), 2008, pp. 374-381.

[77] J. Tang and X. Zhang, "Cross-layer modeling for quality of service guarantees over wireless links," IEEE Transactions on Wireless Communications, vol. 6, no. 12, pp. 4504-4512, 2007.

[78] Y. Fan, F. Su, Y. Li, and H. Xu, "A network-aware scalable video congestion control mechanism in heterogeneous wireless networks," in International Multi-Conference on Computing in the Global Information Technology - (ICCGI'06), 2006, pp. 20-20.

[79] Q. Li and C. N. Georghiades, "Distortion-delay tradeoff for a gaussian source transmitted over a fading channel," in IEEE International Symposium on Information Theory, 2006, pp. 21-25.

[80] A. M. A. Qumi, A. Saiadian, and A. Mirzaee, "Explicit statistical QoS guarantees for video stream over wireless fading channel," in International Conference on Wireless Communications, Networking and Mobile Computing, vol. 2, 2005, pp. 1245-1248.

[81] T. Wang, P. Ma, and Y. Ma, "Adaptive power allocation with qualityof-service guarantees in secure wireless communications," in 9th International Conference on Communications and Networking in China (CHINACOM), 2014, pp. 508-512.

[82] J. Li, N. Bao, W. Xia, and L. Shen, "Adaptive user scheduling and resource management for multiuser MIMO downlink systems with heterogeneous delay requirements," in IEEE Wireless Communications and Networking Conference (WCNC), 2013, pp. 1351-1356.

[83] Q. Du, Y. Huang, P. Ren, and C. Zhang, "Statistical delay control and QoS-driven power allocation over two-hop wireless relay links," in IEEE Global Telecommunications Conference (GLOBECOM), 2011, pp. 1-5.

[84] L. Liu and J.-F. Chamberland, "On the effective capacities of multipleantenna gaussian channels," in IEEE International Symposium on Information Theory (ISIT), 2008, pp. 2583-2587.

[85] L. Zhao, X. Chi, and W. Shi, "A QoS-driven random access algorithm for MPR-capable VLC system," IEEE Communications Letters, vol. 20 , no. 6, pp. 1239-1242, 2016.

[86] E. Morancho, J. M. Llaberia, and A. Olive, "Recovery mechanism for latency misprediction," in International Conference on Parallel Architectures and Compilation Techniques, 2001, pp. 118-128.

[87] J. Zinky, G. Vichniac, and A. Khanna, "Performance of the revised routing metric in the ARPANET and MILNET," in Military Communications Conference MILCOM, 1989, pp. 219-224. 
[88] M. H. Rehmani, M. Reisslein, A. Rachedi, M. Erol-Kantarci, and M. Radenkovic, "Guest editorial special section on smart grid and renewable energy resources: Information and communication technologies with industry perspective," IEEE Transactions on Industrial Informatics, vol. 13, no. 6, pp. 3119-3123, Dec 2017.

[89] M. H. Rehmani, A. Rachedi, M. Erol-Kantarci, M. Radenkovic, and M. Reisslein, "Cognitive radio based smart grid: The future of the traditional electrical grid," Ad Hoc Networks, vol. 41, no. Supplement C, pp. 1 - 4, 2016, cognitive Radio Based Smart Grid The Future of the Traditional Electrical Grid.

[90] H. Wang, Y. Qian, and H. Sharif, "Multimedia communications over cognitive radio networks for smart grid applications," IEEE Wireless Communications, vol. 20, no. 4, pp. 125-132, August 2013.

[91] A. A. Khan, M. H. Rehmani, and M. Reisslein, "Cognitive radio for smart grids: Survey of architectures, spectrum sensing mechanisms, and networking protocols," IEEE Communications Surveys Tutorials, vol. 18, no. 1, pp. 860-898, Firstquarter 2016.

[92] S.-C. Lin and K.-C. Chen, "Cognitive and opportunistic relay for QoS guarantees in machine-to-machine communications," IEEE Transactions on Mobile Computing, vol. 15, no. 3, pp. 599-609, 2016.

[93] Q. Zhu and X. Zhang, "Effective-capacity based auctions for relay selection over wireless cooperative communications networks," in IEEE Global Communications Conference (GLOBECOM), 2016, pp. 1-6.

[94] K. P. Peppas, P. T. Mathiopoulos, and J. Yang, "On the effective capacity of amplify-and-forward multihop transmission over arbitrary and correlated fading channels," IEEE Wireless Communications Letters, vol. 5, no. 3, pp. 248-251, 2016.

[95] W. Yu, L. Musavian, and Q. Ni, "Tradeoff analysis and joint optimization of link-layer energy efficiency and effective capacity toward green communications," IEEE Transactions on Wireless Communications, vol. 15, no. 5, pp. 3339-3353, 2016.

[96] Q. Zhu and X. Zhang, "Effective-capacity based gaming for optimal power and spectrum allocations over big-data virtual wireless networks," in IEEE Global Communications Conference (GLOBECOM), 2015, pp. 1-6.

[97] L. Musavian and Q. Ni, "Effective capacity maximization with statistical delay and effective energy efficiency requirements," IEEE Transactions on Wireless Communications, vol. 14, no. 7, pp. 3824 3835, 2015.

[98] W. Zhou, J. Wang, and S. Li, "Effective capacity of a secondary user without channel side informatuon in nakagami fading channels," in International Conference on Communications, Circuits and Systems (ICCCAS), vol. 1, 2013, pp. 17-20.

[99] F. Benkhelifa, Z. Rezki, and M.-S. Alouini, "Effective capacity of nakagami-m fading channels with full channel state information in the low power regime," in IEEE 24th International Symposium on Personal Indoor and Mobile Radio Communications (PIMRC), 2013, pp. 18831887

[100] S. Aksu and G. K. Kurt, "Effective capacity in multihop multirate adaptive cooperative networks under nakagami-m fading," in IEEE Wireless Communications and Networking Conference (WCNC). IEEE, 2013, pp. 3926-3931

[101] W. Cheng, X. Zhang, and H. Zhang, "Maximizing effective capacity over wireless links under average and peak power constraints," in IEEE International Conference on Communications (ICC), 2012, pp. 51905194.

[102] M. Pirmoradian and C. Politis, "Power allocation in cognitive spectrum sharing area using effective capacity in imperfect fading channel," in International Symposium on Computer Networks and Distributed Systems (CNDS), 2011, pp. 116-121.

[103] L. Musavian and S. Aïssa, "Effective capacity of delay-constrained cognitive radio in nakagami fading channels," IEEE Transactions on Wireless Communications, vol. 9, no. 3, 2010.

[104] S. Ren and K. B. Letaief, "Maximizing the effective capacity for wireless cooperative relay networks with QoS guarantees," IEEE Transactions on Communications, vol. 57, no. 7, 2009.

[105] _ _ "Optimal effective capacity for cooperative relay networks with QoS guarantees," in IEEE International Conference on Communications (ICC), 2008, pp. 3725-3729.

[106] W. Cheng, X. Zhang, and H. Zhang, "Decentralized heterogeneous statistical QoS provisioning for uplinks over 5G wireless networks," in IEEE Global Communications Conference (GLOBECOM), 2016, pp. $1-7$.

[107] J. Wang and X. Zhang, "Statistical QoS-driven resource allocation over FD-SS cooperative cognitive radio networks," in IEEE Global Communications Conference (GLOBECOM), 2016, pp. 1-6.
[108] W. Cheng, X. Zhang, and H. Zhang, "Statistical-QoS driven energyefficiency optimization over green 5G mobile wireless networks," IEEE Journal on Selected Areas in Communications, vol. 34, no. 12, pp. 3092-3107, 2016.

[109] X. Zhang and Q. Zhu, "Information-centric network virtualization for QoS provisioning over software defined wireless networks," in IEEE Military Communications Conference (MILCOM), 2016, pp. 10281033.

[110] L. Ma, Y. Ma, and P. Ma, "Delay-QoS-driven secrecy power allocation in underlay secure cognitive radio system," in IEEE 83rd Vehicular Technology Conference (VTC Spring), 2016, pp. 1-5.

[111] Y. Chen and I. Darwazeh, "An accurate approximation of delay with nakagami-m channels and exponential arrivals," in IEEE Global Communications Conference (GLOBECOM), 2015, pp. 1-6.

[112] Q. Zhu and X. Zhang, "Statistical-QoS based gaming for optimal power allocations over virtualized wireless relay networks supporting multimedia services," in IEEE Military Communications Conference, MILCOM, 2015, pp. 151-156.

[113] X. Zhang, W. Cheng, and H. Zhang, "Heterogeneous statistical QoS provisioning over 5G mobile wireless networks," IEEE Network, vol. 28 , no. 6, pp. 46-53, 2014

[114] L. Musavian and T. Le-Ngoc, "Energy-efficient power allocation over nakagami- $m$ fading channels under delay-outage constraints," IEEE Transactions on Wireless Communications, vol. 13, no. 8, pp. 40814091, 2014.

[115] P. B. Oni, R. Duan, and M. Elmusrati, "Dual analysis of the capacity of spectrum sharing cognitive radio with MRC under nakagami-m fading," in Conference Papers in Science, vol. 2013, 2013.

[116] Y. Wang, P. Ren, and Q. Du, "Statistical QoS driven power allocation for cognitive networks under primary user's outage probability constraint," in IEEE 23rd International Symposium on Personal, Indoor and Mobile Radio Communications - (PIMRC), 2012, pp. 167-172.

[117] L. Musavian and T. Le-Ngoc, "Cross-layer design for cognitive radios with joint amc and ARQ under delay QoS constraint," in IEEE 8th International Wireless Communications and Mobile Computing Conference (IWCMC), 2012, pp. 419-424.

[118] L. Musavian, S. Aissa, and S. Lambotharan, "Adaptive modulation in spectrum-sharing channels under delay quality-of-service constraints," IEEE Transactions on Vehicular Technology, vol. 60, no. 3, pp. 901911, 2011

[119] Y. Ma, D. Yuan, and H. Zhang, "Cross-layer-model based power minimization with quality-of-service guarantees," in IEEE 70th Vehicular Technology Conference Fall, 2009, pp. 1-5.

[120] L. Musavian and S. Aissa, "Adaptive modulation in spectrum-sharing systems with delay constraints," in IEEE International Conference on Communications (ICC), 2009, pp. 1-5.

[121] J. Tang and X. Zhang, "Cross-layer-model based adaptive resource allocation for statistical QoS guarantees in mobile wireless networks,' IEEE Transactions on Wireless Communications, vol. 7, no. 6, 2008.

[122] — "Quality-of-service driven power and rate adaptation over wireless links," IEEE Transactions on Wireless Communications, vol. 6 , no. 8, pp. 3058-3068, 2007.

[123] — "Quality-of-service driven power and rate control in mobile wireless networks," in IEEE International Conference on Communications (ICC), vol. 4, 2006, pp. 1903-1908.

[124] D. Xu and Q. Li, "On the effective capacity region for cognitive radio multiple access channels," AEU-International Journal of Electronics and Communications, vol. 69, no. 6, pp. 958-961, 2015.

[125] U. Celentano and S. Glisic, "Effective capacity of imperfect adaptive wireless communication systems," in IEEE 16th International Symposium on Personal, Indoor and Mobile Radio Communications (PIMRC), vol. 4, 2005, pp. 2191-2195.

[126] H. Sun, Q. Wu, and C. Williamson, "Impact of stochastic traffic characteristics on effective capacity in CDMA networks," in 31st IEEE Conference on Local Computer Networks, 2006, pp. 793-800.

[127] M. You, H. Sun, J. Jiang, and J. Zhang, "Effective rate analysis in weibull fading channels," IEEE Wireless Communications Letters, vol. 5, no. 4, pp. 340-343, 2016.

[128] C. Kabiri, H.-J. Zepernick, and H. Tran, "Effective capacity of cognitive cooperative relay networks over $\alpha-\mu$ fading channels," in 21st AsiaPacific Conference on Communications (APCC), 2015, pp. 427-432.

[129] C. Kabiri, L. Sibomana, and H.-J. Zepernick, "Symbol error probability and effective capacity of an underlay crn over $\alpha-\mu$ fading channels," in 9th International Conference on Signal Processing and Communication Systems (ICSPCS), 2015, pp. 1-6. 
[130] J. Zhang, L. Dai, W. H. Gerstacker, and Z. Wang, "Effective capacity of communication systems over $\kappa-\mu$ shadowed fading channels," Electronics Letters, vol. 51, no. 19, pp. 1540-1542, 2015.

[131] J. Zhang, Z. Tan, H. Wang, Q. Huang, and L. Hanzo, "The effective throughput of MISO systems over k-u fading channels," IEEE Transactions on Vehicular Technology, vol. 63, no. 2, pp. 943-947, 2014.

[132] W. Yu, L. Musavian, and Q. Ni, "Statistical delay QoS driven energy efficiency and effective capacity tradeoff for uplink multi-user multicarrier systems," IEEE Transactions on Communications, 2017.

[133] J. Choi, "Effective capacity of NOMA and a suboptimal power control policy with delay QoS," IEEE Transactions on Communications, 2017.

[134] A. H. Anwar, K. G. Seddik, T. ElBatt, and A. H. Zahran, "Effective capacity of delay-constrained cognitive radio links exploiting primary feedback," IEEE Transactions on Vehicular Technology, vol. 65, no. 9, pp. 7334-7348, 2016.

[135] D. Qiao, "Effective capacity of buffer-aided relay systems with selection relaying," in IEEE Global Communications Conference (GLOBECOM), 2015, pp. 1-7.

[136] Y. Khan, M. Derakhshani, S. Parsaeefard, and T. Le-Ngoc, "Selforganizing TDMA MAC protocol for effective capacity improvement in IEEE 802.11 WLANs," in IEEE Globecom Workshops (GC Wkshps), 2015, pp. 1-6.

[137] Z. Xiao, X. Xie, and S. Zhang, "Effective capacity analysis of link layer based on cognitive multiple access channel," in 5th International Conference on Electronics Information and Emergency Communication (ICEIEC), 2015, pp. 136-141.

[138] W. Yu, L. Musavian, and Q. Ni, "Multi-carrier link-layer energy efficiency and effective capacity tradeoff," in IEEE International Conference on Communication Workshop (ICCW), 2015, pp. 2763-2768.

[139] —, "Weighted tradeoff between effective capacity and energy efficiency," in IEEE International Conference on Communications (ICC), 2015, pp. 238-243.

[140] M. Hammouda, S. Akin, and J. Peissig, "Effective capacity in cognitive radio broadcast channels," in IEEE Global Communications Conference (GLOBECOM), 2014, pp. 1071-1077.

[141] M. M. Butt, A. H. Anwar, A. Mohamed, and T. ElBatt, "Effective capacity of cognitive radio links: Accessing primary feedback erroneously," in 11th International Symposium on Wireless Communications Systems (ISWCS), 2014, pp. 395-400.

[142] R. Li and J. Li, "Effective capacity analysis in underlay cooperative cognitive radio network," in IEEE XXXIth URSI General Assembly and Scientific Symposium (URSI GASS), 2014, pp. 1-4.

[143] X.-Q. Shi and Q.-X. Chu, "Effective capacity of delay quality-ofservice guarantees with imperfect channel information in spectrumsharing environment," in International Conference on Computer Communication and Informatics (ICCCI), 2014, pp. 1-6.

[144] S. Efazati and P. Azmi, "Effective capacity maximization in multirelay networks with a novel cross-layer transmission framework and powerallocation scheme," IEEE transactions on vehicular technology, vol. 63 , no. 4, pp. 1691-1702, 2014.

[145] M. Elalem, "Effective capacity with interference constraints in multichannel spectrum sharing system," in IEEE International Conference on Research and Innovation in Information Systems (ICRIIS), 2013, pp. 404-409.

[146] Y. Yang, H. Ma, and S. Aissa, "Relay selection from an effective capacity perspective," in IEEE 24th International Symposium on Personal Indoor and Mobile Radio Communications (PIMRC), 2013, pp. 10661070 .

[147] W. Cheng, X. Zhang, and H. Zhang, "QoS-aware power allocations for maximizing effective capacity over virtual-MIMO wireless networks," IEEE Journal on Selected Areas in Communications, vol. 31, no. 10, pp. 2043-2057, 2013.

[148] M. Elalem and L. Zhao, "Effective capacity and interference constraints in multichannel cognitive radio network," in IEEE Wireless Communications and Networking Conference (WCNC), 2013, pp. 2993-2998.

[149] M. Lari, A. Mohammadi, A. Abdipour, and I. Lee, "Effective capacity in receive antenna selection and spatially correlated MIMO-OSTBC systems," in IEEE Sixth International Symposium on Telecommunications (IST), 2012, pp. 117-122.

[150] Y. Hu and J. Gross, "On the outage probability and effective capacity of multiple decode-and-forward relay system," in IEEE IFIP Wireless Days (WD), 2012, pp. 1-8.

[151] D. Qiao, M. C. Gursoy, and S. Velipasalar, "The impact of halfduplex relaying on the effective capacity of two-hop communication systems," in 46th Annual Conference on Information Sciences and Systems (CISS), 2012, pp. 1-5.
[152] J. S. Harsini and M. Zorzi, "Effective capacity for multi-rate relay channels with delay constraint exploiting adaptive cooperative diversity," IEEE Transactions on Wireless Communications, vol. 11, no. 9, pp. 3136-3147, 2012.

[153] X. Xie and W. Guo, "Fundamental effective capacity limits of cognitive radio in fading environments with imperfect channel information," in IEEE International Conference on Computational Problem-Solving (ICCP), 2011, pp. 232-235.

[154] D. Qiao, M. C. Gursoy, and S. Velipasalar, "On the effective capacity of two-hop communication systems," in IEEE International Conference on Communications (ICC), 2011, pp. 1-5.

[155] D. Li, "Effective capacity limits of cognitive radio networks under peak interference constraint," in 12th IEEE International Conference on Communication Technology (ICCT), 2010, pp. 218-222.

[156] Y. Xu, J. Wang, and Q. Wu, "Effective capacity region of twouser opportunistic spectrum access in fading channel under discrete transmission rate policy," in International Conference on Wireless Communications and Signal Processing (WCSP), 2010, pp. 1-5.

[157] S. Akin and M. C. Gursoy, "Effective capacity analysis of cognitive radio channels for quality of service provisioning," IEEE Transactions on Wireless Communications, vol. 9, no. 11, pp. 3354-3364, 2010.

[158] L. Musavian, S. Aïssa, and S. Lambotharan, "Effective capacity for interference and delay constrained cognitive radio relay channels," IEEE Transactions on Wireless Communications, vol. 9, no. 5, 2010.

[159] A. Balasubramanian and S. L. Miller, "The effective capacity of a time division downlink scheduling system," IEEE Transactions on Communications, vol. 58, no. 1, 2010.

[160] G. Femenias, J. Ramis, and L. Carrasco, "Using two-dimensional markov models and the effective-capacity approach for cross-layer design in AMC/ARQ-based wireless networks," IEEE transactions on vehicular technology, vol. 58, no. 8, pp. 4193-4203, 2009.

[161] T. Abrão, L. D. H. Sampaio, S. Yang, K. T. K. Cheung, P. J. E. Jeszensky, and L. Hanzo, "Energy efficient OFDMA networks maintaining statistical QoS guarantees for delay-sensitive traffic," IEEE Access, vol. 4, pp. 774-791, 2016.

[162] M. Sinaie, A. Zappone, E. A. Jorswieck, and P. Azmi, "A novel power consumption model for effective energy efficiency in wireless networks," IEEE Wireless Communications Letters, vol. 5, no. 2, pp. 152-155, 2016.

[163] M. Ozmen and M. C. Gursoy, "Secure transmission of delay-sensitive data over wireless fading channels," IEEE Transactions on Information Forensics and Security, 2017.

[164] M. Qin, Q. Yang, J. Yang, K.-s. Kwak, and R. R. Rao, “Throughput of QoS guaranteed wireless systems with/without channel state information," IEEE Transactions on Vehicular Technology, 2016.

[165] D. Qiao and M. C. Gursoy, "Statistical delay tradeoffs in bufferaided two-hop wireless communication systems," IEEE Transactions on Communications, vol. 64, no. 11, pp. 4563-4577, 2016.

[166] Y. Li, M. C. Gursoy, and S. Velipasalar, "Joint mode selection and resource allocation for D2D communications under queueing constraints," in IEEE Conference on Computer Communications Workshops (INFOCOM WKSHPS), 2016, pp. 490-495.

[167] S. Efazati and P. Azmi, "Cross layer power allocation for selection relaying and incremental relaying protocols over single relay networks," IEEE Transactions on Wireless Communications, vol. 15, no. 7, pp. 4598-4610, 2016.

[168] X. Mi, L. Xiao, M. Zhao, X. Xu, S. Zhou, and J. Wang, "Heterogeneous statistical QoS-driven power control for D2D communications underlaying cellular networks," in 23rd International Conference on Telecommunications (ICT), 2016, pp. 1-5.

[169] A. Sharifian, R. Schoenen, and H. Yanikomeroglu, "Joint realtime and nonrealtime flows packet scheduling and resource block allocation in wireless OFDMA networks," IEEE Transactions on Vehicular Technology, vol. 65, no. 4, pp. 2589-2607, 2016.

[170] D. Qiao, "Achievable rate of two-hop channels under statistical delay constraints," in IEEE Global Communications Conference (GLOBECOM), 2015, pp. 1-6.

[171] Z. Li, Z. Lu, X. Wen, W. Jing, Z. Zhang, and F. Fu, "Distributed power control for two-tier femtocell networks with QoS provisioning based on Q-learning," in IEEE 82nd Vehicular Technology Conference (VTC Fall), 2015, pp. 1-6.

[172] Y. Wang, P. Ren, Q. Du, and L. Sun, "Optimal power allocation for underlay-based cognitive radio networks with primary user's statistical delay QoS provisioning," IEEE Transactions on Wireless Communications, vol. 14, no. 12, pp. 6896-6910, 2015. 
[173] S. Akin and M. Fidler, "Backlog and delay reasoning in HARQ system," in 27th International Teletraffic Congress (ITC 27), 2015, pp. $185-193$.

[174] K. T. Phan and T. Le-Ngoc, "Adaptive link selection in bufferaided relaying with statistical QoS constraints," in IEEE International Conference on Communications (ICC), 2015, pp. 1813-1818.

[175] Y. Li, L. Liu, H. Li, J. Zhang, and Y. Yi, "Resource allocation for delaysensitive traffic over LTE-advanced relay networks," IEEE Transactions on Wireless Communications, vol. 14, no. 8, pp. 4291-4303, 2015.

[176] C. She, C. Yang, and L. Liu, "Energy-efficient resource allocation of wireless systems with statistical QoS requirement," in IEEE Online Conference on Green Communications (OnlineGreencomm), 2014, pp. $1-6$.

[177] Y. Wang and K. R. Liu, "Statistical delay QoS protection for primary users in cooperative cognitive radio networks," IEEE Communications Letters, vol. 19, no. 5, pp. 835-838, 2015.

[178] S. Efazati and P. Azmi, "Statistical quality of service provisioning in multi-user centralised networks," IET Communications, vol. 9, no. 5, pp. 621-629, 2015.

[179] F. Fu, Z. Lu, X. Wen, W. Jing, Z. Zhang, and Z. Li, "Exact potential game based power control with QoS provisioning in two-tier femtocell networks," in International Symposium on Wireless Personal Multimedia Communications (WPMC), 2014, pp. 180-185.

[180] P. Zabihi, M. F. Sabahi, and K. Shahtalebi, "Power allocation game considering QoS and interference for fading multiple access channels," in 7th International Symposium on Telecommunications (IST), 2014, pp. 1166-1171.

[181] D. Xu and Q. Li, "Resource allocation in delay-QoS constrained multiuser cognitive radio networks," in Sixth International Conference on Wireless Communications and Signal Processing (WCSP), 2014, pp. $1-6$.

[182] W. Jing, Z. Lu, Z. Zhang, H. Zhang, and X. Wen, "Energy-efficient power allocation with QoS provisioning in OFDMA femtocell networks," in IEEE Wireless Communications and Networking Conference (WCNC), 2014, pp. 1473-1478.

[183] A. Helmy and T. Le-Ngoc, "Low-complexity QoS-aware frequency provisioning in downlink multi-user multicarrier systems," in IEEE Wireless Communications and Networking Conference (WCNC), 2014, pp. 1785-1790.

[184] L. Musavian and Q. Ni, "Delay-QoS-driven spectrum and energy efficiency tradeoff," in IEEE International Conference on Communications (ICC), 2014, pp. 4981-4986.

[185] W. Jing, Z. Lu, H. Zhang, Z. Zhang, J. Zhao, and X. Wen, "Energysaving resource allocation scheme with QoS provisioning in OFDMA femtocell networks," in IEEE International Conference on Communications Workshops (ICC), 2014, pp. 912-917.

[186] Z. Zhang, X. Wen, Z. Li, S. He, W. Jing, and J. Zhao, "QoS-aware energy-efficient power control in two-tier femtocell networks based on Q-learning," in IEEE 21st International Conference on Telecommunications (ICT), 2014, pp. 313-317.

[187] S. Khakurel, T. Le-Ngoc, and L. Musavian, "QoS-driven energyefficient power adaptation in a multi-channel fading communication link," in IEEE Global Communications Conference (GLOBECOM), 2013, pp. 4038-4043.

[188] W. Jiao, M. Sheng, Y. Zhang, and K.-S. Lui, "On end-to-end delay of multi-hop wireless networks," in IEEE 77th Vehicular Technology Conference (VTC Spring), 2013, pp. 1-5.

[189] A. Helmy, L. Musavian, and T. Le-Ngoc, "Energy-efficient power allocation for multicarrier systems with delay-outage probability constraints," in IEEE International Conference on Communications (ICC). IEEE, 2013, pp. 5872-5877.

[190] Y. Li, L. Liu, H. Li, Y. Li, and Y. Yi, "Adaptive resource allocation for heterogeneous traffic over heterogeneous relay networks," in IEEE International Conference on Communications (ICC), 2013, pp. 54315436

[191] A. Helmy, L. Musavian, and T. Le-Ngoc, "Energy-efficient power adaptation over a frequency-selective fading channel with delay and power constraints," IEEE Transactions on Wireless Communications, vol. 12, no. 9, pp. 4529-4541, 2013.

[192] C. Xiong, G. Y. Li, Y. Liu, Y. Chen, and S. Xu, "Energy-efficient design for downlink OFDMA with delay-sensitive traffic," IEEE Transactions on Wireless Communications, vol. 12, no. 6, pp. 3085-3095, 2013.

[193] L. Musavian and T. Le-Ngoc, "Energy-efficient power allocation for delay-constrained systems," in IEEE Global Communications Conference (GLOBECOM), 2012, pp. 3554-3559.

[194] J. Yuan and Q. Wang, "Delay quality-of-service driven resource allocation for relay-based OFDMA cognitive radio networks," in IEEE
Global Communications Conference (GLOBECOM), 2012, pp. 39943999.

[195] A. Gopalakrishna and D. B. Ha, "Capacity analysis of cognitive radio relay networks with interference power constraints in fading channels," in International Conference on Computing, Management and Telecommunications (ComManTel), 2013, pp. 111-116.

[196] C. Lin, Y. Liu, and M. Tao, "Cross-layer resource allocation of two-way relaying for statistical delay-QoS guarantees," in IEEE International Conference on Communications (ICC), 2012, pp. 4741-4745.

[197] J. Yuan and Q. Wang, "Delay quality-of-service driven resource allocation for relay-based multiuser OFDMA networks," in IEEE International Conference on Communications (ICC), 2012, pp. 38893894.

[198] D. Chen, H. Ji, and V. C. M. Leung, "Cross-layer QoS provisioning for cooperative transmissions over cognitive radio relay networks with imperfect spectrum sensing," in IEEE Global Telecommunications Conference (GLOBECOM), 2011, pp. 1-5.

[199] M. Taki and F. Lahouti, "Link adaptation design for interfering cognitive radio with QoS constraints," in 17th International Conference on Telecommunications, 2010, pp. 435-441.

[200] N. Gunaseelan, L. Liu, J. F. Chamberland, and G. H. Huff, "Performance analysis of wireless hybrid-ARQ systems with delay-sensitive traffic," IEEE Transactions on Communications, vol. 58, no. 4, pp. 1262-1272, 2010.

[201] D. Qiao, M. C. Gursoy, and S. Velipasalar, "Analysis of energy efficiency in fading channels under QoS constraints," in IEEE Global Telecommunications Conference (GLOBECOM), 2008, pp. 1-5.

[202] L. Musavian and S. Aissa, "Quality-of-service based power allocation in spectrum-sharing channels," in IEEE Global Telecommunications Conference (GLOBECOM ), 2008, pp. 1-5.

[203] Q. Dai, Y. Zhao, and J. Zhao, "A resource allocation strategy using FDMA over wireless relay networks," in 4th International Conference on Wireless Communications, Networking and Mobile Computing, 2008, pp. 1-5.

[204] L. Liu, J. F. Chamberland, and K. Qaraqe, "Enabling delay-sensitive applications over sensor networks using a two-tier architecture and multi-antenna cluster heads," in IEEE International Workshop on Computational Advances in Multi-Sensor Adaptive Processing, 2007, pp. 301-304.

[205] J. Tang and X. Zhang, "Quality-of-service driven power and rate adaptation for multichannel communications over wireless links," IEEE Transactions on Wireless Communications, vol. 6, no. 12, pp. 43494360, 2007.

[206] L. Xie and X. Zhang, "Cross-layer resource allocation strategies for quality-of-service driven opportunistic routing over wireless relay networks," in Annual Conference on Information Sciences and Systems, 2007, pp. 7-12.

[207] _ "Opportunistic cooperation for quality of service provisionings over wireless relay networks," in IEEE International Conference on Communications (ICC), 2007, pp. 254-259.

[208] — "TDMA and FDMA based resource allocations for quality of service provisioning over wireless relay networks," in IEEE Wireless Communications and Networking Conference, 2007, pp. 3153-3157.

[209] J. Tang and X. Zhang, "Cross-layer resource allocation over wireless relay networks for quality of service provisioning," IEEE Journal on Selected Areas in Communications, vol. 25, no. 4, pp. 645-656, 2007.

[210] L. Liu, P. Parag, J. Tang, W. Y. Chen, and J. F. Chamberland, "Resource allocation and quality of service evaluation for wireless communication systems using fluid models," IEEE Transactions on Information Theory, vol. 53, no. 5, pp. 1767-1777, 2007.

[211] J. Chen and Y. Yang, "WSN12-4: multi-hop delay performance in wireless mesh networks," in IEEE Global Telecommunications Conference (GLOBECOM ), 2006, pp. 1-5.

[212] J. Tang and X. Zhang, "QoS-driven power and rate adaptation for multicarrier communications over mobile wireless networks," in 40th Annual Conference on Information Sciences and Systems, 2006, pp. 321-326.

[213] _ , "QoS-driven adaptive power and rate allocation for multichannel communications in mobile wireless networks," in IEEE International Symposium on Information Theory, 2006, pp. 2516-2520.

[214] D. Park and B. G. Lee, "QoS support by using CDF-based wireless packet scheduling in fading channels," IEEE Transactions on Cоттиnications, vol. 54, no. 11, pp. 2051-2061, 2006.

[215] X. Zhang, J. Tang, H.-H. Chen, S. Ci, and M. Guizani, "Cross-layerbased modeling for quality of service guarantees in mobile wireless networks," IEEE Communications Magazine, vol. 44, no. 1, pp. 100106, 2006. 
[216] S. Ci, M. Guizani, and G. B. Brahim, "A dynamic resource allocation scheme for delay-constrained multimedia services in CDMA 1 times;EV-DV forward link," IEEE Journal on Selected Areas in Communications, vol. 24, no. 1, pp. 46-53, 2006.

[217] M. Bourouha, S. Ci, G. B. Brahim, and M. Guizani, "A cross-layer design for QoS support in the 3GPP2 wireless systems," in IEEE Global Telecommunications Conference Workshops (GlobeCom Workshops), 2004, pp. 56-61.

[218] D. Wu and R. Negi, "A wireless channel model for support of quality of service," in IEEE Global Telecommunications Conference (GLOBECOM), vol. 1, 2001, pp. 695-699.

[219] L. Carrasco, J. Ramis, and G. Femenias, "Multidimensional markov models for the cross-layer design of multi-rate wireless systems using the effective capacity function," in IEEE 69th Vehicular Technology Conference (VTC), 2009, pp. 1-5.

[220] M. Z. Hassan, M. J. Hossain, J. Cheng, and V. C. Leung, "Effective capacity of coherent POLMUX OWC impaired by atmospheric turbulence and pointing errors," Journal of Lightwave Technology, vol. 34, no. 21, pp. 5007-5022, 2016.

[221] M. Z. Hassan, V. C. Leung, M. J. Hossain, and J. Cheng, "Statistical delay aware joint power allocations and relay selection for NLOS multichannel OWC," in IEEE Global Communications Conference (GLOBECOM), 2016, pp. 1-6.

[222] _ "QoS-aware joint power allocations and relay selection for NLOS coherent optical wireless communications," in IEEE International Conference on Communications (ICC), 2016, pp. 1-7.

[223] — "QoS-aware and energy-aware adaptive power allocations for coherent optical wireless communications," in IEEE International Conference on Communications (ICC), 2016, pp. 1-7.

[224] — , "Effective capacity performance of coherent POLMUX OWC with power adaptation," in IEEE Global Communications Conference (GLOBECOM), 2015, pp. 1-7.

[225] M. Elalem and L. Zhao, "Effective capacity optimization based on overlay cognitive radio network in gamma fading environment," in IEEE Wireless Communications and Networking Conference (WCNC), 2013, pp. 2999-3004.

[226] P. Larsson, J. Gross, H. Al-Zubaidy, L. K. Rasmussen, and M. Skoglund, "Effective capacity of retransmission schemes: A recurrence relation approach," IEEE Transactions on Communications, vol. 64, no. 11, pp. 4817-4835, 2016.

[227] Q. Du and X. Zhang, "Effective capacity optimization with layered transmission for multicast in wireless networks," in International Wireless Communications and Mobile Computing Conference (IWCMC), 2008, pp. 267-272.

[228] — , "Effective capacity of superposition coding based mobile multicast in wireless networks," in IEEE International Conference on Communications (ICC), 2009, pp. 1-5.

[229] K. T. Phan, T. Le-Ngoc, and L. B. Le, "Optimal resource allocation for buffer-aided relaying with statistical QoS constraint," IEEE Transactions on Communications, vol. 64, no. 3, pp. 959-972, 2016.

[230] D. Qiao, M. C. Gursoy, and S. Velipasalar, "Effective capacity region and optimal power control for fading broadcast channels," in IEEE International Symposium on Information Theory Proceedings (ISIT), 2011, pp. 2974-2978.

[231] M. C. Gursoy, D. Qiao, and S. Velipasalar, "Analysis of energy efficiency in fading channels under QoS constraints," IEEE Transactions on Wireless Communications, vol. 8, no. 8, pp. 4252-4263, 2009.

[232] S. Vassaki, A. D. Panagopoulos, and P. Constantinou, "Effective capacity and optimal power allocation for mobile satellite systems and services," IEEE Communications Letters, vol. 16, no. 1, pp. 60-63, 2012.

[233] Z. Ji, C. Dong, Y. Wang, and J. Lu, "On the analysis of effective capacity over generalized fading channels," in IEEE International Conference on Communications (ICC), 2014, pp. 1977-1983.

[234] Y. Yang, "Effective capacity of a novel spectrum-band selection scheme in spectrum-sharing networks," IEEE Transactions on Vehicular Technology, vol. 66, no. 3, pp. 2838-2843, 2017.

[235] Y. Yang, S. Aïssa, and K. N. Salama, "Spectrum band selection in delay-QoS constrained cognitive radio networks," IEEE Transactions on Vehicular Technology, vol. 64, no. 7, pp. 2925-2937, 2015.

[236] D. Xu, Z. Feng, Y. Wang, and P. Zhang, "Capacity of cognitive radio under delay quality-of-service constraints with outdated channel feedback," in IEEE 23rd International Symposium on Personal Indoor and Mobile Radio Communications (PIMRC), 2012, pp. 1704-1709.

[237] M. Elalem and L. Zhao, "Effective capacity optimization for cognitive radio network based on underlay scheme in gamma fading channels," in International Conference on Computing, Networking and Communications (ICNC), 2013, pp. 714-718.

[238] S. Xiangqun and C. Qingxin, "Effective capacity of cognitive radio systems in asymmetric fading channels," The Journal of China Universities of Posts and Telecommunications, vol. 22, no. 3, pp. 18-25, 2015.

[239] D. Li, "Effect of channel estimation errors on arbitrary transmit antenna selection for cognitive MISO systems," IEEE Communications Letters, vol. 15, no. 6, pp. 656-658, 2011.

[240] G. Zhang, A. Huang, H. Shan, J. Wang, T. Q. Quek, and Y.-D. Yao, "Design and analysis of distributed hopping-based channel access in multi-channel cognitive radio systems with delay constraints," IEEE Journal on Selected Areas in Communications, vol. 32, no. 11, pp. 2026-2038, 2014

[241] G. Zhang, A. Huang, J. Wang, H. Shan, and T. Q. Quek, "Hoppingbased channel access in cognitive radio systems," in IEEE 77th Vehicular Technology Conference (VTC Spring), 2013, pp. 1-5.

[242] P. Zhang, G. Lv, and C. Zhang, "Optimal power allocation for delayconstrained multiple-channel cognitive radio systems," in International Wireless Communications and Mobile Computing Conference (IWCMC), 2014, pp. 352-356.

[243] M. Cui, C. Sun, P. Ma, and Y. Ma, "Joint resource allocation with statistical delay QoS guarantees for spectrum leasing CR networks,' in 8th International Conference on Wireless Communications \& Signal Processing (WCSP), 2016, pp. 1-5.

[244] Y. Ma, P. Ma, X. Yang, Q. Liu, and L. Ma, "Resource allocation with delay QoS guarantees for spectrum sharing in cognitive radio networks," in IEEE 26th Annual International Symposium on Personal, Indoor, and Mobile Radio Communications (PIMRC), 2015, pp. 645649.

[245] M. A. Ramirez-Reyna, F. A. Cruz-Pérez, M. E. Rivero-Angeles, and G. Hernandez-Valdez, "Dynamic spectrum leasing strategies for coordinated cognitive radio networks with delay-tolerant traffic," in IEEE 25th Annual International Symposium on Personal, Indoor, and Mobile Radio Communication (PIMRC), 2014, pp. 1200-1205.

[246] S. Gunawardena and W. Zhuang, "Capacity analysis and call admission control in distributed cognitive radio networks," IEEE Transactions on Wireless Communications, vol. 10, no. 9, pp. 3110-3120, 2011.

[247] _ " "Voice capacity of cognitive radio networks," in IEEE International Conference on Communications, 2010, pp. 1-5.

[248] A. Abdrabou and W. Zhuang, "Statistical QoS evaluation for cognitive radio networks," in IEEE Global Telecommunications Conference (GLOBECOM), 2011, pp. 1-5.

[249] Y. Saleem and M. H. Rehmani, "Primary radio user activity models for cognitive radio networks: A survey," Journal of Network and Computer Applications, vol. 43, no. Supplement C, pp. 1 - 16, 2014.

[250] M. Amjad, F. Akhtar, M. H. Rehmani, M. Reisslein, and T. Umer, "Full-duplex communication in cognitive radio networks: A survey," IEEE Communications Surveys Tutorials, vol. 19, no. 4, pp. 21582191, 2017.

[251] F. Akhtar, M. H. Rehmani, and M. Reisslein, "White space: Definitional perspectives and their role in exploiting spectrum opportunities,' Telecommunications Policy, vol. 40, no. 4, pp. 319 - 331, 2016

[252] Q. Wu, Y. Huang, J. Wang, and Y. Cheng, "Effective capacity of cognitive radio systems with GSC diversity under imperfect channel knowledge," IEEE Communications Letters, vol. 16, no. 11, pp. 1792$1795,2012$.

[253] S. Vakilinia and I. Vakilinia, "QoS aware energy efficient resource allocation in wireless cooperative OFDMA relay networks," in IEEE 6th Joint IFIP Wireless and Mobile Networking Conference (WMNC), 2013, pp. 1-4.

[254] G. Ozcan and M. C. Gursoy, "Effective capacity analysis of fixedgain and variable-gain af two-way relaying," in IEEE 78th Vehicular Technology Conference (VTC Fall), 2013, pp. 1-5.

[255] S. Efazati and P. Azmi, "Effective capacity analysis of three node relay networks with adaptive relaying protocols," in IEEE Sixth International Symposium on Telecommunications (IST), 2012, pp. 194-199.

[256] K. T. Phan and T. Le-Ngoc, "Effective capacities of dual-hop networks with relay selection," in IEEE Wireless Communications and Networking Conference (WCNC), 2014, pp. $2014-2019$.

[257] — , "Effective capacity of dual-hop networks with a concurrent buffer-aided relaying protocol," in IEEE International Conference on Communications (ICC), 2014, pp. 1048-1052.

[258] D. Qiao, "Fixed versus selective scheduling for buffer-aided diamond relay systems under statistical delay constraints," IEEE Transactions on Communications, 2017. 
[259] — - "On the achievable throughput of buffer-aided diamond relay systems under delay constraints," in IEEE Global Communications Conference (GLOBECOM), 2016, pp. 1-7.

[260] Q. Yuan, Y. Hu, H. Zhi, and M. He, "Cross-layer power allocation scheme for two-way amplify-and-forward relaying system," in IEEE International Conference on Communication Software and Networks (ICCSN), 2016, pp. 381-386.

[261] D. Wu and R. Negi, "Effective capacity channel model for frequencyselective fading channels," Wireless Networks, vol. 13, no. 3, pp. 299$310,2007$.

[262] B. Shariati, J. M. Rivas-Moscoso, D. Klonidis, I. Tomkos, S. BenEzra, F. Jiménez, D. M. Marom, P. S. Khodashenas, J. Comellas, and L. Velasco, "Options for cost-effective capacity upgrades in backbone optical networks," in 21st European Conference on Networks and Optical Communications (NOC), 2016, pp. 35-40.

[263] L. Chen and W. Wang, "Effective capacity of MIMO free-space optical systems over gamma-gamma turbulence channels," Optics Communications, vol. 382, pp. 450-454, 2017.

[264] A. Huang, O. Kabranov, and D. Makrakis, "Performance analysis of a novel optical network architecture - PetaWeb," in International Conference on Communication Technology Proceedings ( ICCT), vol. 1, 2003, pp. 81-85.

[265] A. Huang, T. Yang, O. Kabranov, and D. Makrakis, "QoS performance in IP over PetaWeb optical network," in IEEE International Conference on Communications, Circuits and Systems and West Sino Expositions, vol. 1, 2002, pp. 664-668.

[266] A. Davy, B. Meskill, and J. Domingo-Pascual, "An empirical study of effective capacity throughputs in 802.11 wireless networks," in IEEE Global Communications Conference (GLOBECOM), 2012, pp. 17701775 .

[267] A. Abdrabou and W. Zhuang, "Statistical QoS routing for IEEE 802.11 multihop ad hoc networks," IEEE Transactions on Wireless Communications, vol. 8, no. 3, pp. 1542-1552, 2009.

[268] — , "Statistical call admission control for IEEE 802.11 multi-hop wireless ad hoc networks," in IEEE Global Telecommunications Conference (GLOBECOM), 2008, pp. 1-5.

[269] _ _ "Stochastic delay guarantees and statistical call admission control for IEEE 802.11 single-hop ad hoc networks," IEEE Transactions on Wireless Communications, vol. 7, no. 10, pp. 3972-3981, 2008.

[270] E. Kafetzakis, K. Kontovasilis, and I. Stavrakakis, "A novel effective capacity-based framework for providing statistical QoS guarantees in IEEE 802.11 WLANs," Computer Communications, vol. 35, no. 2, pp. 249-262, 2012.

[271] — "Effective-capacity-based stochastic delay guarantees for systems with time-varying servers, with an application to IEEE 802.11 WLANs," Performance Evaluation, vol. 68, no. 7, pp. 614-628, 2011.

[272] B. Rashid and M. H. Rehmani, "Applications of wireless sensor networks for urban areas: A survey," Journal of Network and Computer Applications, vol. 60, no. Supplement C, pp. 192 - 219, 2016.

[273] M. Amjad, M. Sharif, M. K. Afzal, and S. W. Kim, "TinyOS-new trends, comparative views, and supported sensing applications: A review," IEEE Sensors Journal, vol. 16, no. 9, pp. 2865-2889, 2016.

[274] M. Amjad, M. K. Afzal, T. Umer, and B.-S. Kim, "QoS-aware and heterogeneously clustered routing protocol for wireless sensor networks," IEEE Access, vol. 5, pp. 10 250-10 262, 2017.

[275] T. Umer, M. Amjad, M. K. Afzal, and M. Aslam, "Hybrid rapid response routing approach for delay-sensitive data in hospital body area sensor network," in Proceedings of the 7th International Conference on Computing Communication and Networking Technologies, 2016, p. 3.

[276] Y. Chen and I. Darwazeh, "A sampling method for predicting end-toend delay performance in wireless sensor networks," in 1st International Conference on $5 G$ for Ubiquitous Connectivity (5GU). IEEE, 2014, pp. 279-284.

[277] M. Amjad, A. Ahmad, M. H. Rehmani, and T. Umer, "A review of EVs charging: From the perspective of energy optimization, optimization approaches, and charging techniques," Transportation Research Part D: Transport and Environment, vol. 62, pp. 386-417, 2018.

[278] T. Umer, M. Amjad, N. Shah, and Z. Ding, "Modeling vehicles mobility for connectivity analysis in vanet," in Intelligent Transportation Systems. Springer, 2016, pp. 221-239.

[279] A. Abdrabou and W. Zhuang, "On a stochastic delay bound for disrupted vehicle-to-infrastructure communication with random traffic," in IEEE Global Telecommunications Conference (GLOBECOM ), 2009, pp. 1-6.

[280] — "Probabilistic delay control and road side unit placement for vehicular ad hoc networks with disrupted connectivity," IEEE Journal on Selected Areas in Communications, vol. 29, no. 1, pp. 129-139, 2011.

[281] C. Guo, L. Liang, and G. Y. Li, "Resource allocation for low-latency vehicular communications: An effective capacity perspective," IEEE Journal on Selected Areas in Communications, vol. 37, no. 4, pp. 905917, 2019.

[282] M. Hughes and V. M. Jovanovic, "Small cells-effective capacity relief option for heterogeneous networks," in IEEE Vehicular Technology Conference (VTC Fall), 2012, pp. 1-6.

[283] Z. Huang, H. Xia, Z. Zeng, and Y. Liu, "Optimization of energy efficiency for ofdma femtocell networks based on effective capacity," in IEEE Vehicular Technology Conference (VTC Fall), 2012, pp. 1-5.

[284] S. He, L. Zhang, X. Wen, Z. Zhang, Z. Lu, and Y. Sun, "Pricebased power control with statistical delay QoS guarantee in twotier femtocell networks," in IEEE 21st International Conference on Telecommunications (ICT), 2014, pp. 318-322.

[285] C. Lin, M. Tao, G. Stuber, and Y. Liu, "Distributed cross-layer resource allocation for statistical QoS provisioning in femtocell networks," in IEEE International Conference on Communications (ICC), 2013, pp. 5000-5004.

[286] A. A. Khan, M. H. Rehmani, and A. Rachedi, "Cognitive-radiobased internet of things: Applications, architectures, spectrum related functionalities, and future research directions," IEEE Wireless Communications, vol. 24, no. 3, pp. 17-25, 2017.

[287] P. Popovski, J. J. Nielsen, C. Stefanovic, E. de Carvalho, E. Ström, K. F. Trillingsgaard, A.-S. Bana, D. M. Kim, R. Kotaba, J. Park et al., "Ultra-reliable low-latency communication (URLLC): principles and building blocks," arXiv preprint arXiv:1708.07862, 2017.

[288] A. H. Solana, A. V. Bardajf, and F. C. Palacio, "Uplink call admission control techniques for multimedia packet transmission in UMTS WCDMA system," in 5th European Personal Mobile Communications Conference, 2003, pp. 209-213.

[289] A. H. Solana, A. V. Bardaji, and F. C. Palacio, "Capacity analysis and performance evaluation of call admission control for multimedia packet transmission in UMTS WCDMA system," in IEEE Wireless Communications and Networking (WCNC), vol. 3, 2003, pp. 15501555.

[290] S. Liang, P. Ma, and Y. Ma, "Delay-QoS-driven robust power allocation over full-duplex relay multicarrier secure system," in IEEE International Conference on Communication Systems (ICCS), 2016, pp. 1-5.

[291] S. Lee, D. Kim, H. Lee, S. Weon, and D. Hong, "FDR and HDR for AF relaying with QoS guarantees," in 18th IEEE International Symposium on Consumer Electronics (ISCE 2014), 2014, pp. 1-2.

[292] D. Qiao, M. C. Gursoy, and S. Velipasalar, "Effective capacity of two-hop wireless communication systems," IEEE Transactions on Information Theory, vol. 59, no. 2, pp. 873-885, 2013.

[293] M. He, Y. Hu, H. Zhi, and Q. Yuan, "Cross-layer power allocation scheme for wireless full duplex relaying system," in 8th IEEE International Conference on Communication Software and Networks (ICCSN), 2016, pp. 375-380.

[294] Y. Li, M. C. Gursoy, and S. Velipasalar, "Throughput and mode selection in two-way MIMO systems under queuing constraints," in IEEE International Conference on Communications (ICC), 2015, pp. 2271-2276.

[295] A. Sadeghi, M. Luvisotto, F. Lahouti, S. Vitturi, and M. Zorzi, "Statistical QoS analysis of full duplex and half duplex heterogeneous cellular networks," in IEEE International Conference on Communications (ICC), 2016, pp. 1-6.

[296] J. Choi, "On large deviations of HARQ with incremental redundancy over fading channels," IEEE Communications Letters, vol. 16, no. 6 , pp. 913-916, 2012.

[297] D. Qiao, "Outage effective capacity of buffer-aided diamond relay systems using harq-ir," IEEE Transactions on Vehicular Technology, vol. 68 , no. 1, pp. 540-553, Jan 2019.

[298] M. Amjad and L. Musavian, "Performance analysis of NOMA for ultra-reliable and low-latency communications," arXiv preprint arXiv:1810.02276, 2018.

[299] J. Choi, "An effective capacity-based approach to multi-channel lowlatency wireless communications," IEEE Transactions on Communications, vol. 67, no. 3, pp. 2476-2486, 2019.

[300] J. Chen and K.-K. Wong, "Improving energy efficiency for multiuser MIMO systems with effective capacity constraints," in IEEE 69th Vehicular Technology Conference (VTC-Spring), 2009, pp. 1-5.

[301] T. A. Zewde and M. C. Gursoy, "QoS-driven resource allocation for SWIPT with finite-alphabet inputs," in IEEE Wireless Communications and Networking Conference (WCNC), 2017, pp. 1-6. 
[302] — , "Energy-efficient time allocation for wireless energy harvesting communication networks," in IEEE Globecom Workshops (GC Wkshps), 2016, pp. 1-6.

[303] F. Akhtar and M. H. Rehmani, "Energy replenishment using renewable and traditional energy resources for sustainable wireless sensor networks: A review," Renewable and Sustainable Energy Reviews, vol. 45, no. Supplement C, pp. $769-784,2015$.

[304] A. Davy and J. D. Pascual, "CrEST: an effective capacity estimation tool for wireless networks," in IEEE IFIP Wireless Days (WD), 2013, pp. 1-7.

[305] M. Vasef, "Effective capacity of a rayleigh fading channel in the presence of interference," arXiv preprint arXiv:1207.2904, 2012.

[306] Q. Cui, Y. Gu, W. Ni, and R. P. Liu, "Effective capacity of licensedassisted access in unlicensed spectrum: From theory to applications," IEEE Journal on Selected Areas in Communications, 2017.

[307] J. Li, Y. Ding, Q. Ye, N. Zhang, and W. Zhuang, "On effective capacity and effective energy efficiency in relay-assisted wireless networks," IEEE Transactions on Vehicular Technology, vol. 67, no. 5, pp. 43894400, 2018. 